\title{
Zur chemischen Erkenntnis biologischer Oxydationsreaktionen.
}

Von

C. Engler und R. O. Herzog.

(Aus dem chemischen Institut der technischen Hochschule zu Karlsruhe.)

(Der Redaktion zugegangen am 8. März 1909.)

\section{Theoretisches.}

Historische Vorbemerkungen.

Man weiß seit der Entdeckung des Sauerstoffs, daß er sich als solcher an den chemischen Vorgängen beteiligt, die sich im Organismus abspielen. Anderseits ist auch bald erkannt worden, daß zwischen der Verbrennung, die sich unter Feuererscheinung abspielt, und gewissen langsam verlaufenden Prozessen nur Unterschiede bezüglich Temperatur und Geschwindigkeit bestehen; so kam man dazu, die chemische Wirkung des Sauerstoffs auf die Organismen zu den *langsamen Verbrennungen» zu zählen.

Priestley, ${ }^{1}$ ) Crawford ${ }^{2}$ ) und besonders Lavoisier ${ }^{3}$ ) haben sich naturgemäß mit der Feststellung begnügt, daß die Wirkung des Sauerstoffs auf die Organismen der bei der Verbrennung völlig gleicht. In den Chemismus dieser Oxydationen hat zuerst Schönbein ${ }^{4}$ ) einzudringen versucht. Er beobachtete 1840 bei der langsamen Oxydation des feuchten Phosphors das Auftreten von Ozon; Sauerstoff war also in eine «aktivere» Form übergeführt worden. Gewisse unstreitig sehr feine Be-

1) Phil. Trans., Bd. LXVI, S. 220; s. a. Jörgensen, Entdeckung des Sauerstoffs, 1909, S. 58.

2) Journ. de Physiol., 1782, Bd. XV.

s) Mém. de l'Acad. pr. 1777, S. 185; s. a. Euvres, Bd. II, S. 174; ferner Abh. mit Laplace, das., S. 283, S. 724; auch 0 stwalds Klassiker, Nr. 40. - Die alte Literatur s. bei J. Müller, Physiologie, 1833, Bd. I, S. 316. - Über den Einfluß Liebigs auf die Entwicklung vgl. Volhard. J. v. Liebig, Bd. II, S. 120.

4) Diese und die folgenden Angaben, die in diesem Abschnitt nicht ausdrücklich angeführt sind, finden sich ausführlich in Engler und Weissberg, «Kritische Studien zur Autoxydation», Braunschweig 1904, 
obachtungen führten ihn zur Annahme einer zweiten aktiven Form des Sauerstoffs, des Antozons. In diesen beiden «aktiven» Formen sollte also der Sauerstoff als Oxydationsmittel wirken können. Brodie, Meissner, Glausius traten weiterhin für diese Theorie ein; Weltzien, Berthelot, Houzeau, Wurtz u. a. lehnten sie ab. Durch den von Engler und Nasse, sowie von Engler und Wild definitiv erbrachten Nachweis, daß Antozon nicht existiere, war ihr von chemischer Seite der Boden entzogen. Von biologischer Seite war der Einwand zu machen, daß das Vorkommen von Ozon und ozonbildenden Stoffen im Organismus u. a. gerade auch nach Versuchen Schönbeins unerwiesen ${ }^{1}$ ) und auch wegen der Giftigkeit des Ozons unwahrscheinlich sei.

Schönbein ${ }^{2}$ ) hatte zuerst nur angenommen, daß im Blute - ebenso wie er es in den Pilzen nachgewiesen hatte eine Materie vorhanden sei, «durch deren Vermittlung die Oxydation sowohl des Blutes selbst als auch tierischer Organe herbeigeführt werde». Erst später ${ }^{3}$ ) wendet er seine Theorie der Oxydation durch Zustandsänderung des Sauerstoffs in Ozon und Antozon an, nachdem ihn auch eingehende gerade physiologischchemische Versuche darin bestärkt haben. A. Schmidt ${ }^{4}$ ) erklärte die biologische Oxydationswirkung des eingeatmeten Sauerstoffgases sehr ähnlich wie Schönbein durch die Annahme, daß es im Blut in der Form von Ozon wirksam sei. Aus den bereits erwähnten Gründen entstanden noch verschiedene andere Versuche zur Erklärung der biologischen Oxydation.

weiterhin zit. als E. und W. - Es darf auch nicht unterlassen werden, die Quellen zahlreicher Angaben in der vorliegenden Studie anzuführen; so seien genannt: Kobert, «Lehrbuch der Intoxikationens, Bd. II; Heffter, in Ergebn. der Physiologie; Czapek, Biochemie d. Pflanzen; L af ars Handb. d. techn. Mykologie, bes. Behrens, Bd. I. S. 668; ferner d. Lehrbücher d. physiol. Chem. u. a. Leider konnten gewisse Angaben in der Originalliteratur nicht eingesehen werden.

1) Vgl. Huizinga, Virch ow s Arch., Bd. XLII, S. 359 (1908), Nasse, Pflügers Archiv, Bd. III, S. 205 (1870).

₹) Basler Verh., Bd. I, S. 339 (1855). - Journ. f. prakt. Chem., Bd. LXVII, S. 496 (1856).

3) Basler Verh., Bd. III, S. 516 (1863); vgl. auch Sitzungsber. der

Kgl. Bayer. Akad. d. Wiss., München 1863, Bd. I, S. 274.

4) Über Ozon im Blut, Dorpat 1862. 
Naegeli ${ }^{1}$ ) hat die molekulare «Schwingungstheorie» auf die biologische Oxydation übertragen; die «spezifischen Bewegungszustände in dem lebenden Plasma» sollen auf den Sauerstoff übertragen werden. «Erreicht die Störung des Gleichgewichtes in den Molekülen einen gewissen Grad, so tritt mit Hilfe der chemischen Affinität die Umsetzung ein.» Mehr Anklang fand Hoppe-Seyler. ${ }^{2}$ ) Er hält dafür, daß reduzierende Stoffe das Sauerstoffmolekül in Atome zersprengen und das eine Atom aufnehmen. Damit wird das zweite im status nascens frei und somit zur Oxydationswirkung auch schwer oxydablen Stoffen gegenüber besonders befähigt. Ihm schließt sich Baumann $^{3}$ ) an. Nencki und Sieber ${ }^{4}$ ) glauben, daß die Oxydation eine Funktion des lebenden Protoplasmas sei. Dabei weist aber Nencki ${ }^{5}$ ) mehrmals auf Radziszewski ${ }^{6}$ ) hin, der angegeben hatte, daß Alkali indifferenten atmosphärischen Sauerstoff in seine Atome zu spalten vermöge. Schmiedeberg ${ }^{7}$ ) erklärt, daß in den Geweben Bedingungen gegeben sind, die aus gewissen Paarlingen Synthesen unter Wasserauftritt bewirken, wie aus Benzoesäure und Glykokoll Hippursäure, aus Schwefelsäure und Phenol die Ätherschwefelsäure. «Die Oxydation des Benzylalkohols erfolgt fast genau in derselben Weise. Sie kann als eine Synthese angesehen werden zwischen zwei Paarlingen, von denen der eine $\mathrm{O}_{2}$ ist. Auch hier treten die Reste nach Abgabe des Wassers zu einer neuen Verbindung, der Benzoesäure, zusammen.» ${ }^{8}$ )

Ein anderer Gedanke wurde von M. Traube ${ }^{9}$ ) ausgesprochen. Er nimmt an, daß das ganze Sauerstoffmolekül das

1) Theorie der Gärung, S. 43 (1879).

2) Diese Zeitschrift, Bd. II, S. 1 (1878-79).

$\left.{ }^{3}\right)$ Diese Zeitschrift, Bd. V, S. 244 (1884).

4) Journ. f. prakt. Chem., Bd. XXVI, S. 1 (1882).

5) Journ. f. prakt. Chem., Bd. XXIII, S. 87 (1881); Pflügers Arch., Bd. XXXI, S. 319 (1883) u. a. a. 0.

6) Ann. Chem. Pharm., Bd. CCIII, S. 305 (1880).

7) Arch. f. exp. Path. u. Pharm., Bd. XIV, S. 288 (1881).

8) Arch. f. exp. Path. u. Pharm., Bd. XIV, S. 298.

9) B. B., Bd. XV, S. 222, 659, 2421 (1882); Bd. XVI, S. 123, 463 (1883); Bd. XVIII, S. 1877, 1887, 1890, 1894 (1885); Bd. XIX, S. 1115 (1886); Bd. XXII, S. 1496 (1889); Bd. XXVI, S. 1471, 1476 (1893). 
Wirksame sei, in der Weise, daß das (fast immer notwendige) Wasser gleichzeitig zu Wasserstoffsuperoxyd oxydiert werde. Z. B.

$$
\mathrm{Zn}+2 \mathrm{H}_{2} \mathrm{O}+\mathrm{O}_{2}=\mathrm{Zn}(\mathrm{OH})_{2}+\mathrm{H}_{2} \mathrm{O}_{2} \text {. }
$$

Diese Aufnahme von Sauerstoff hat er als Autoxydation bezeichnet. Dieselbe Anschauung etwa vertritt auch Nef. Unter den Pflanzenphysiologen ist J. Reinke ${ }^{1}$ ) für die Traubesche Theorie eingetreten. In seinen bedeutungsvollen «Beiträgen zur Kenntnis der Oxydationsvorgänge hat $\mathrm{Pfeffer}^{2}$ ) vor allem darauf hingewiesen, daß «erst durch Einbeziehung des zu veratmenden Körpers in den Stoffumsatz die geeigneten Verbindungen oder Bedingungen geschaffen werden », welche zur Oxydation führen.

Ungefähr gleichzeitig und unabhängig von einander haben Engler ${ }^{3}$ ) und $\mathrm{Bach}^{4}$ ) auf dem Grundgedanken Traubes eine neue, umfassende Theorie entwickelt und ihr auch experimentelle Grundlagen gegeben, zu deren Vertiefung ferner Bodländer, Manchot, Luther u. a. wichtige Beiträge geliefert haben. Die Grundannahme ist, daß der erste Schritt bei der langsamen Verbrennung, in der Anlagerung des - ungesättigten Sauerstoffmoleküls an andere - ebenfalls ungesättigte - Stoffe besteht; die sich dabei bildenden, superoxydartigen Additionsprodukte werden nach dem Vorschlage von Engler und Weissberg Moloxyde genannt.

Es sei endlich noch erwähnt, daß auch die elektrochemischen Theorien, die von Clausius im Anschluß an Schönbein, von Richarz, R. v. Helmholtz und Richarz und von van't Hoff im AnschluB an Hoppe-Seyler entwickelt wurden, eine abschließende Darstellung durch Haber ${ }^{5}$ ) fanden, welcher ihnen eine Form gab, die sich inhaltlich völlig mit der oben gegebenen Autoxydationstheorie deckt.

1) Bot. Zg., Bd. XLI, S. 66, 90 (1883).

2) Abh. Kgl. Sächs. Ges. d. Wiss., Bd. XV, S. 373 (1890).

$\left.{ }^{3}\right)$ Verh. d. naturw. Vereins Karlsruhe, 20. XI. 1896, Bd. XIII, S. 72. Engler und Wild, B. B., Bd. XXX, S. 1669 (1897).

4) C. R., Bd. CXXIV, S. 951 (1897).

5) Z. f. physikal. Chem., Bd. XXXIV, S. 513 (1900); Bd. XXXV, S. 81, 609 (1900). - Physikal. Z., Bd. I, S. 419 (1900). - Z. f. Elektrochem., Bd. VII, S. 441 (1901). 
Das Ergebnis dieser kurzen historischen Übersicht ist also, daß zurzeit eine Theorie besteht, die sich mit der Einwirkung des gasförmigen Sauerstoffs auf andere Stoffe beschäftigt. In der vorliegenden Studie wird untersucht werden, wie weit sie eine Erklärung für die Vorgänge im Organismus zu erbringen vermag. Das setzt voraus, festgestellt $z u$ haben, wie weit überhaupt Erklärungen zu Oxydationsvorgängen zu geben sind. Im folgenden wird also zunächst eine Systematik der Oxydationsvorgänge entwickelt.

\section{Direkte und indirekte Autoxydation.}

Nach der Autoxydationstheorie wirkt der Sauerstoff auf die a u toxydablen Stoffe, indem er sich als ${ }_{-0}^{-0}$ anlagert; erfolgt dies direkt, so spricht man von direkter Autoxydation. Als autoxydabel mögen vorläufig die additionsfähigen Stoffe angesprochen werden. Beispiele sind die Bildung von $\mathrm{RbO}_{2}$ und $\mathrm{H}_{2} \mathrm{O}_{2}$ bei der «Verbrennung * von $\mathrm{Rb}$ und $\mathrm{H}$, die Bildung von Peroxyden aus Phosphinen, ungesättigten Kohlenwasserstoffen, Aldehyden und anderen Stoffen. ${ }^{1}$ ) Es wurde bereits erwähnt, daß die Additionsprodukte von $\mathrm{O}_{2}$ und den autoxydablen Stoffen, welche auch als Autoxydatoren bezeichnet werden, als Moloxyde angesprochen werden. Sie enthalten den Sauerstoff als Atompaar, also molekular gebunden: $\mathrm{R}\left\langle{ }_{0}^{0}\right.$. Natürlich soll aber nicht gesagt sein, daß die Moloxyde immer auch die allererste Form der Additionsprodukte darstellen.

Viel häufiger als die direkte Autoxydation ist die indirekte. In diesen Fällen ist der Stoff, der die Autoxydation bewirkt, nicht auch zugleich derselbe, der den molekularen Sauerstoff bindet, sondern er macht einen andern Stoff, häufig H-Ionen zu Autoxydatoren, die zum Unterschied von dem bereits definierten als indirekte (oder auch sekundäre) Autoxydatoren bezeichnet werden. Den Stoff, der die Bildung von indirekten

1) Genaueres s. bei Engler und Weissberg, l. c. 
Autoxydatoren bewirkt, nennt man Pseudoautoxydator. Ein Beispiel dieser Art ist

$$
\begin{aligned}
& \text { Pseudo- Indir. Moloxyd } \\
& \text { autoxy- Autoxy- } \\
& \text { dator dator } \\
& \mathrm{Zn}+\underset{\mathrm{OH}}{\mathrm{OH}}+\underset{-\mathrm{O}}{\mathrm{O}} \rightarrow \mathrm{Zn}^{-0}{ }_{\mathrm{OH}}^{\mathrm{OH}}+\stackrel{\mathrm{H}-\mathrm{O}}{\mathrm{H}-\mathrm{O}}
\end{aligned}
$$

Man sieht, daß in diesem Fall der Pseudoautoxydator gleichfalls eine chemische Veränderung erleidet (wie im angeführten Fall besteht sie sehr häufig zunächst in der Aufnahme von $\mathrm{OH}$, in anderen Fällen bleibt als Oxydationsprodukt ein Spaltrest zurück, wie im folgenden Beispiel Indigo).

Eine ganze Reihe von Kohlenstoffverbindungen dissoziiert sehr leicht Wasserstoffatome $a b$, und gerade sie spielen darum zumal in wässeriger Lösung die Rolle von Pseudoautoxydatoren. Hierher gehören z. B. die Leukoverbindungen der Farbbasen, Hydrazoverbindungen, Phenole (Alkohole), Amine u. a. Als Beispiel diene

$$
\begin{aligned}
& \text { Pseudoautoxydator Indirekter Moloxyd } \\
& \text { Indigweiß } \\
& \text { Autoxydator } \\
& \text { Indigo } \nearrow_{\mathrm{H}}^{\mathrm{H}}+\underset{\mathrm{OH}}{\mathrm{OH}} \mathrm{H}+\underset{-\mathrm{O}}{\mathrm{H}} \rightarrow \text { Indigo }+\underset{\mathrm{H}-\mathrm{O}}{\mathrm{O}}+2 \mathrm{H}_{2} \mathrm{O}
\end{aligned}
$$

Man sieht, daß die folgende Schreibweise

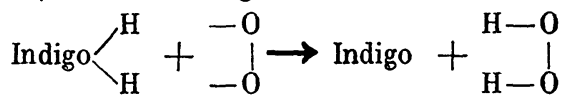

den Tatbestand leicht verdeckt - gerade weil man den Prozeß leicht in solcher Weise auffaßt, wurde eben der Name Pseudoautoxydator gewählt - , denn just die Ionen des Wassers sind für das Zustandekommen der Reaktion wesentlich. Und gerade weil es sich in der Regel um Ionenreaktionen handelt, verlaufen auch die indirekten Autoxydationen meistens schnell im Gegensatz zu den direkten Autoxydationen. ${ }^{1}$ )

Das bisher Erörterte zeigt also, daß der gasförmige Sauerstoff als solcher nur auf Stoffe einwirken kann, die als direkte oder indirekte Autoxydatoren bezeichnet werden, ferner bei

1) Nebenbei sei erwähnt, daß die Traubesche Theorie im wesentlichen die indirekte Autoxydation im Auge hat. 
Gegenwart letzterer noch auf Pseudoautoxydatoren. Aber gerade die normalen Nahrungsmittel resp. ihre ersten Spaltungsprodukte, Fettsäuren und Glycerin aus dem Fett, die weitaus meisten Aminosäuren aus den Proteinen, die Kohlehydrate, gehören nicht zu den bereits behandelten Gruppen. Nun ließe sich natürlich die bisher öfters erörterte, aber meist unerwiesene Annahme machen, daß diese Spaltungsprodukte weiter in Bruchstücke zertrümmert würden, die autoxydabel oder Pseudoautoxydatoren sind. Das mag vielleicht auch gelegentlich der Fall sein. Man kann aber zu viel allgemeineren Erklärungen aus den bisherigen chemischen Erfahrungen gelangen, wie weiter angedeutet werden wird. Welche Erklärungen zu Recht bestehen, ist nur experimentell zu entscheiden.

Umwandlungen der Moloxyde.

Schreiben wir die bisher besprochenen Fälle schematisch, so wurde allein erörtert $(A=$ direkter oderindirekter Autoxydator $)$ :

$$
\mathrm{A}+\mathrm{O}_{2} \rightarrow \mathrm{AO}_{2} \text {. }
$$

Es sei hier gleich darauf hingewiesen, daß diese Gleichung natürlich nicht stets quantitativ im Sinne des Pfeiles verlaufen muß, sondern es kann ein Gleichgewicht bestehen, wie gewöhnlich geschrieben:

$$
\mathrm{A}+\mathrm{O}_{2} \rightleftarrows \mathrm{AO}_{\mathbf{2}} \text {. }
$$

Das ist jedenfalls zwischen Hämoglobin, Sauerstoff und Oxyhämoglobin der Fall, ${ }^{1}$ ) wenn auch die Verhältnisse im speziellen noch komplizierter liegen können. So ist möglich, "daß wir mit einem Gemisch von Blutfarbstoffen mit verschiedener Sauerstoffbindung zu schaffen haben». ${ }^{2}$ )

Es kann also in diesem Falle vom Moloxyd auch wieder der ganze Sauerstoff abgegeben werden; ebenso ist das einem zweiten Autoxydator gegenüber möglich. Ferner kann die Reaktion eintreten:

$$
2 \mathrm{AO}_{2} \rightarrow 2 \mathrm{AO}+\mathrm{O}_{2} \text {, }
$$

1) Vgl. Hüfner, Arch. f. Anat. u. Physiol., 1890, S. 1, 1901 Suppl., S. 187. - Ghr. Bohr,Zentralblatt f. Physiol., Bd. XVII, S. 682 (1904). - V. Henri, C. R. Soc. biol., Bd. LVI, S. 339.

2) Nagels Handb. d. Physiol., Bd. II, S. 96 (1905); s. a. Zentralblatt f. Physiol., Bd. IV, S. 249 (1890). 
wie bei der Zersetzung von Wasserstoffsuperoyd in Wasser und $\mathrm{O}_{2}$. Weiter sind noch einige Möglichkeiten zu besprechen, wie die Moloxyde weiterhin umgewandelt werden können.

So können sich die Moloxyde umlagern, polymerisieren (wie Aldehydperoxyde) usw. Besonders ist auch noch die Bildung von sekundären Superoxyden von Wichtigkeit, die eintritt, wenn vorhandene Stoffe mit dem gebildeten Moloxyd reagieren und das Reaktionsprodukt ein neues Superoxyd vorstellt. Bekannte Reaktionen dieser Art sind die teilweise und unvollständige Verseifung von Moloxyden:

a)<smiles>O[14CH2]OO</smiles>

sekundäres Peroxyd.

(Beispiel: Terpentinölhydroperoxyd).

b)

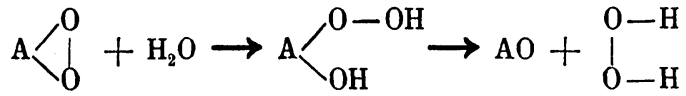
sekundäres Peroxyd.

(Beispiele sind die überaus häufigen Fälle der sekundären Bildung von $\mathrm{H}_{2} \mathrm{O}_{2}$ bei der indirekten Autoxydation.)

Statt Wasser können auch andere Oxyde an solchen Reaktionen teilnehmen (Beispiel: Acetylbenzoylsuperoxyd bei der Autoxydation von Benzaldehyd in Gegenwart von Essigsäureanhydrid); ferner kann eine Anhydridbildung der Peroxyde eintreten (Beispiel: Cerperoxyd aus $\mathrm{H}_{2} \mathrm{O}_{2}$ und niederen Oxydationsstufen von Cer).

Sehr häufig sind die Fälle, daß die Reaktionen in der entgegengesetzten Richtung, wie angegeben, verlaufen, also z. B.

$$
\begin{aligned}
& \mathrm{A}=\mathrm{O}+\mathrm{H}_{2} \mathrm{O}_{2} \rightarrow \mathrm{A} \backslash_{\mathrm{OH}}^{\mathrm{O}-\mathrm{OH}} \rightarrow \mathrm{A}_{\mathrm{O}} \backslash_{\mathrm{O}}^{\mathrm{O}}+\mathrm{H}_{2} \mathrm{O} \\
& \text { sekundäres } \\
& \text { Peroxyd } \\
& \begin{array}{c}
\text { oder } \mathrm{A}-\mathrm{OH}+\mathrm{H}_{2} \mathrm{O}_{2} \rightarrow \underset{\text { sekundäres }}{\text { Peroxyd }} \\
\text { Aeroxy }
\end{array}
\end{aligned}
$$

oder reversibel sind.

So verläuft die Wirkung von $\mathrm{H}_{2} \mathrm{O}_{2}$ auf Ferrosulfat, und auf der Bildung des sekundären Peroxyds beruht seine $0 x y-$ 
dationswirkung. ${ }^{1}$ ) Ganz ebenso ist die Wirkung von $\mathrm{H}_{2} \mathrm{O}_{2}$ auf Peroxydase, resp. von Oxygenase auf Peroxydase anzunehmen.

\section{Acceptoren.}

Kehren wir zur Gleichung I (S. 333) zurück, so bestehen weiterhin zunächst folgende Möglichkeiten: Erstens, es kann ein dritter Stoff $B$ vorhanden sein, der zwar nicht autoxydabel ist, aber von $\mathrm{AO}_{2}$ oxydiert werden kann, welches häufig ein höheres «Oxydationspotential» als $\mathrm{O}_{2}$ besitzt, öfters aber wohl auch nur deswegen wirksam ist, weil ein anderer, schnellerer Reaktionsweg eingeschlagen wird, z. B. über ein Additionsprodukt. Man nennt den Stoff B Acceptor. Es tritt also z. B. sehr häufig die Folgereaktion ein:

$$
\mathrm{AO}_{2}+\mathrm{B} \rightarrow \mathrm{AO}+\mathrm{BO} \text {. }
$$

Das Moloxyd hat den Sauerstoff «hälftig» abgegeben; der Autoxydator ist selbst oxydiert worden. (Beispiele: Autoxydation des Terpentinöls, des Benzaldehyds u. a. bei Gegenwart von Indigo, der Cerosalze bei Gegenwart von Arsenit.)

Zweitens, der autoxydable Stoff A kann auch Selbstacceptor sein, z. B. bei der Verbrennung des Wasserstoffs

$$
\begin{aligned}
\mathrm{H}_{2}+\mathrm{O}_{2} & \longrightarrow \mathrm{H}_{2} \mathrm{O}_{2} \\
\mathrm{H}_{2} \mathrm{O}_{2}+\mathrm{H}_{8} & \longrightarrow 2 \mathrm{H}_{2} \mathrm{O} .
\end{aligned}
$$

Überhaupt kann jeder Autoxydator zum Acceptor werden, wenn neben ihm ein zweiter, schneller autoxydierender Stoff vorhanden ist; dieser überträgt ihm dann den aufgenommenen Sauerstoff. Hier spielt die Reaktionsgeschwindigkeit eine entscheidende Rolle. Die Reaktionsgeschwindigkeiten der Vorgänge entscheiden auch, ob man bei der Sauerstoffabgabe der Moloxyde an Acceptoren die ersteren überhaupt beobachten kann; verläuft die Superoxydbildung schneller als die Oxydation des Acceptors, so wird es möglich sein, im entgegengesetzten Falle nicht. ${ }^{2}$ )

1) Auch die begünstigende Wirkung der Alkalien bei Oxydationsvorgängen könnte aus der Bildung von Alkaliperoxyden oder m. a. W. der (mehr als $\mathrm{H}_{2} \mathrm{O}_{2}$ dissoziierten) Salze von Hydroperoxyd herrühren.

2) «Es hängt nur von der relativen Geschwindigkeit des Verlaufes der beiden Reaktionen ab, ob man das Superoxyd beobachten kann oder nicht. Verläuft der primäre Prozeß (Superoxyḍbildung) rascher als der sekundäre (weitere Oxydationswirkung), so wird man, wie beim 
Ob ein Stoff einem Peroxyd, überhaupt einem Oxydationsmittel gegenüber als Acceptor zu wirken vermag, ist wahrscheinlich wesentlich eine Funktion von seiner Additionsfähigkeit an das Oxydans. Welcher Stoff als Oxydationsmittel auftritt, hängt natürlich davon $\mathrm{ab}, \mathrm{ob}$ das oder die zunächst entstehenden Oxydationsprodukte gegenüber dem Oxydàtionsmittel stabil sind oder nicht; im letzten Fall schreitet die Oxydation eben bis zur Bildung beständiger Verbrennungsprodukte weiter.

Katalytische, induzierte und Folgereaktionen.

Wirkt das bei der Folgereaktion gebildete 0xyd des Autoxydators noch oxydierend oder, anders ausgedrückt, vermag es vom Acceptor weiter reduziert zu werden, so kann der Autoxydator regeneriert werden, wie bei der Autoxydation der Cero- oder Ferrosalze bei Gegenwart von Glukose als Acceptor, bei der Platinkatalyse:

$$
\mathrm{AO}+\mathrm{B} \rightarrow \mathrm{A}+\mathrm{BO} .
$$

A spielt dann also die Rolle eines Katalysators, dessen Teilnahme in der Reaktionsgleichung

nicht erkannt wird.

$$
2 \mathrm{~B}+\mathrm{O}_{2} \rightarrow 2 \mathrm{BO}
$$

Die Wirkung der Katalasen hat man sich aber anders vorzustellen. Sie gehören höchstwahrscheinlich zu den Stoffen, die mit $\mathrm{H}_{2} \mathrm{O}_{2}$ sekundäre Peroxyde geben, welche dann weiter reagieren; also etwa:

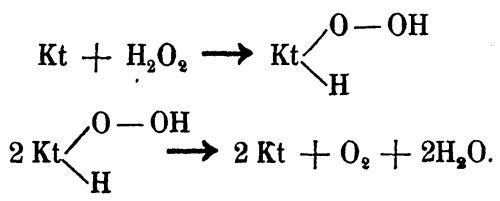

Ein schönes Beispiel einer sehr ähnlich verlaufenden Reaktion haben Bredig und v. Antrop off ${ }^{1}$ ) kürzlich kennen gelehrt:

$$
2 \mathrm{H}_{2} \mathrm{O}_{2}+\mathrm{Hg}=2 \mathrm{H}_{2} \mathrm{O}+\mathrm{HgO}_{2} \text {. }
$$

Terpentinöl bei gewöhnlicher Temperatur, das Superoxyd wahrnehmen, im andern Fall - z. B. beim Terpentinöl bei höherer Temperatur oder beim Triäthylphosphin bei gewöhnlicher Temperatur - entgeht das rasch wieder verschwindende Superoxyd unserer Beobachtung.) C. Engler, B. B., Bd. XXXII, S. 3100 (1900).

i) Z. f. Elektrochem., Bd. XII, S. 585 (1906). 
Tritt die Folgereaktion:

$$
\mathrm{HgO}_{2}=\mathrm{Hg}+\mathrm{O}_{2}
$$

ein, dann ist der Autoxydator wiederum regeneriert, wie das in der Tat geschieht. ${ }^{1}$ )

Der oben besprochene Fall

$$
\mathrm{AO}_{2}+\mathrm{B} \rightarrow \mathrm{AO}+\mathrm{BO}
$$

ist besonders noch von Interesse, wenn beim Fehlen von $B$ die ebenfalls erwähnte Reaktion

$$
\mathrm{AO}_{\mathbf{z}}+\mathrm{A} \longrightarrow 2 \mathrm{AO}
$$

eintritt. Es liegt dann ein Spezialfall der sogenannten induzierten oder gekoppelten Reaktionen ${ }^{2}$ ) vor.

Einmal findet $\mathrm{zwischen} \mathrm{A}$ und $\mathrm{O}_{2}$ die Reaktion statt

$$
\begin{gathered}
\mathrm{A}+\mathrm{O}_{2} \longrightarrow \mathrm{AO}_{2} \\
\mathrm{AO}_{2}+\mathrm{A} \longrightarrow 2 \mathrm{AO}
\end{gathered}
$$

während (bei Abwesenheit von A) $\mathrm{B}$ mit $\mathrm{O}_{2}$ nicht reagiert. Anderseits bilden sich, wenn gleichzeitig $\mathrm{A}, \mathrm{B}$ und $\mathrm{O}_{2}$ vorhanden sind, sowohl AO wie BO (freilich spielt dabei die Geschwindigkeit der Vorgänge eine erhebliche Rolle). ${ }^{3}$ ) Man nennt dann die Reaktion $2 \mathrm{~A}+\mathrm{O}_{2} \longrightarrow 2 \mathrm{AO}$ die induzierende, die Reaktion $2 \mathrm{~B}+\mathrm{O}_{2} \longrightarrow 2 \mathrm{BO}$ die induzierte.

Seien ganz allgemein M, N, P drei Stoffe, welche in folgender Weise reaktionsfähig sind:

$$
\begin{gathered}
M+N=(M N) \text { reagieren }(+) \\
P+N=\text { reagieren nicht }(\mathcal{P}) \\
M+N+P=(M N)+(P N) \text { reagieren }(+)
\end{gathered}
$$

1) Bredig und Weinmayr, Z. f. physikal. Chem., Bd. XLII, S. 601 (1903) und Bredig und Wilke, Verh. d. naturwiss.-med. Ver. Heidelberg, N. F., Bd. VIII, S. 165 (1905).

2) Über Stufenreaktionen im allgemeinen vgl. Ostwald, ,Lehrbuch d. allgem. Chem.s, Bd. II, S. 2; Wegscheider, Z. f. physikal. Chem., Bd. XXXV, S. 513 (1900); Bd. XXXIX, S. 257 (1902); Kaufler, das., Bd. LV, S. 502 (1906); Abel, das., Bd. LVI, S. 558 (1906); Rakowski, das., Bd. LVII, S. 321 (1906); Federlin, das., Bd. XLI, S. 565 (1903); Schilow, das., Bd. XLII, S. 641 (1903); Brunner, dass., Bd. LII, S. 89 (1905); Skrabal, «Die induzierten Reaktionen॰ (1908), das. auch weitere ausführlichere Literatur.

$\left.{ }^{3}\right)$ Darauf hat Engler öfters hingewiesen, z. B.: B. B. Bd. XXXII, S. 3100 (1900). - Vgl. auch Skrabal, 1. c. 
(wobei die eingeklammerten Buchstaben, die miteinander in Reaktion getretenen Stoffe bezeichnen), dann hat man es mit induzierten oder gekoppelten Reaktionen zu tun.

Luther und Schilow ${ }^{1}$ ) haben folgende Nomenklatur eingeführt: $\mathrm{N}$, der in beiden Reaktionen vorkommende Stoff, heißt der Aktor (in unserem Fall $\mathrm{O}_{2}$ ), $\mathrm{M}$ der Induktor (Autoxydator), $\mathrm{P}$ der Acceptor. ${ }^{2}$ )

Die induzierten Reaktionen scheinen außerordentlich häufig vorzukommen (sind also auch jedenfalls im Organismus sehr verbreitet); insbesondere kennt man viele gekoppelte OxydationsReduktions-Reaktionen.

Es sei hier die interessante Angabe von Abelous und Alois ${ }^{3}$ ) angeführt, daß es im Tier- und Pflanzenreich Stoffe

1) Z. f. physikal. Chem., Bd. XLVI, S. 77 (1903).

2) Die letztere Bezeichnung könnte vielleicht zu Mißverständnissen führen. Nach der von Engler und Weissberg gewählten Nomenklatur heißt Acceptor nur, wie oben auseinandergesetzt, der vom Peroxyd atomistischen Sauerstoff aufnehmende Stoff. Luthers und Schilows Klassifikation ist natürlich auf jede Art Reaktionen anwendbar, z. B. auch auf Prozesse, bei denen nicht der Aktor ein Oxydationsmittel und Induktor und Acceptor die reduzierenden Stoffe sind, sondern umgekehrt der Aktor ein Reduktionsmittel, während Induktor und «Acceptor > oxydierend wirken.

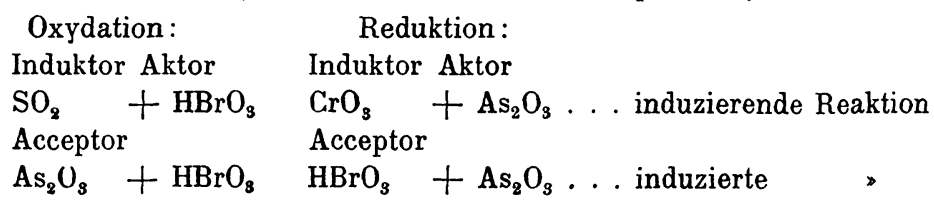

Also nur für den auf S. 337 angeführten Fall, wenn es sich um Autoxydation handelt, decken sich beide Bezeichnungsweisen, für andere gekoppelte Reaktionspaare (wie z. B. ein Peroxyd oder ein anderes 0xydationsmittel den Induktor vorstellen kann) ist das Luther-Schilow sche Schema allgemein:

$$
\begin{aligned}
& \text { Aktor + Induktor }=+ \\
& \text { Aktor + Acceptor }=0 \\
& \text { Aktor + Induktor }=+ \\
& \text { Aktor + Acceptor }=+
\end{aligned}
$$

Bezüglich weiterer Fälle sei auf Skrabal, 1. c. S. 9 u. ff. verwiesen. (In obigen Gleichungen bedeutet + reagieren, 0 reagieren nicht.)

3) C. R., Bd. CXXXVIII, S. 382 (1904). - Die Stoffe werden wohl ohne genügenden Beweis als Enzyme bezeichnet. - Vgl. übrigens auch Diese Zeitschrift, Bd. LVIII, S. 538 (1909). 
gebe, die gleichzeitig reduzierend und oxydierend wirkten; so werde von ihnen Salizylaldehyd zur Salizylsäure oxydiert, doch nur im Beisein von Kaliumchlorat und ähnlichen sauerstoffreichen Verbindungen, die gleichzeitig reduziert werden.

Nach Versuchen, die von R. O. Herzog mit A. Meier und A. Polotzky ausgeführt wurden, scheinen auch die $0 x y-$ dasereaktionen hierher zugehören. Die Oxydasen (Oxygenase + Peroxydase) würden dann den Induktor, Sauerstoff den Aktor und die Oxydasereagenzien die Acceptoren darstellen.

Anderseits ergibt sich gerade aus dem Diskutierten, wie nahe katalytische und Induktionsvorgänge einander liegen, besonders bei den Oxydationsreaktionen; denn tritt z. B. neben der induzierenden Reaktion

$$
\mathrm{AO}_{2}+\mathrm{A} \rightarrow 2 \mathrm{AO}
$$

und der induzierten Reaktion

die Reaktion

$$
2 \mathrm{~B}+\mathrm{O}_{2} \rightarrow 2 \mathrm{BO}
$$

$$
\mathrm{AO}+\mathrm{B} \rightarrow \mathrm{A}+\mathrm{BO}
$$

ein, so hängt es bloß von dem Verhältnis der Geschwindigkeiten der Reaktionen $a b$, ob man eine Katalyse oder eine Induktion beobachtet. Aus diesen Gründen sind auch früher von Engler und Weißberg beide Erscheinungen gemeinsam unter dem Titel Autoxykatalyse behandelt worden.

Ferner zeigt auch wohl schon das soeben Behandelte die außerordentliche Bedeutung der als Folgereaktionen bezeichneten Vorgänge. Je nachdem, ob ein oder mehrere bestimmte Stoffe vorhanden sind, spielen sich chemische Prozesse in verschiedener Richtung und mit oft enorm verschiedener Geschwindigkeit ab. Dies kann darin seine Ursache haben, daß ein hemmendes Reaktionsprodukt (das zum Beispiel das Gleichgewicht der aktiven Stoffe beeinflußt) gebunden wird, oder in irgend welchen ähnlichen Gründen, die man allgemein als Erzeugung oder Verhinderung von Reaktionshemmungen und -beschleunigungen beschreibt, die aber natürlich in allen speziellen Fällen einzeln untersucht werden müssen. Nur auf einen Fall, der sehr häufig wiederkehrt, sei hier hingewiesen, 
nämlich auf die Wirkung von Alkalien; sehr häufig sind sie Ursache von hierher gehörigen Prozessen. ${ }^{1}$ )

Auf die vielseitigen Möglichkeiten gegenseitiger Reaktionsbeeinflussung will wohl auch M. Jakoby ${ }^{2}$ ) hinweisen, wenn er dem komplizierten Stoffgemisch im Organismus eine gewisse Rolle für das Zustandekommen oder Verhindertwerden chemischer Vorgänge zuschreibt und vom Einfluß des «Milieus» auf den Chemismus spricht.

Aus dem Voranstehenden ergibt sich, wie weit bisher eine Theorie der Oxydationsprozesse gegeben werden kann.

Die Einwirkung des gasförmigen Sauerstoffs auf Verbindungen, die Autoxydation, läßt sich weitgehend analysieren. Das Wesentliche des Vorgangs ist durch die Theorie klargestellt und es gelingt leicht, bestimmte Fälle den gegebenen Schematen unterzuordnen. Dagegen sind die weiteren Oxydationsvorgänge in ihrem Verlauf sehr kompliziert und in erster Linie von bestimmten qualitativen Vorbedingungen abhängig, für die eine mehr oder weniger vollständige Systematik noch fehlt. Nur gewisse Faktoren sind bisher erkannt.

Bei der Übersicht über eine größere Anzahl von Fällen, wie sie im weiteren gegeben werden soll, ist jedenfalls einerseits die Wahrscheinlichkeit vorhanden, daß wenigstens eine gewisse Registrierung gelingt, und anderseits möge gezeigt werden, nach welcher Richtung allgemeine Grundlagen gegeben oder zu suchen und spezielle Fragen zu lösen sind.

\section{Spezielle Fälle.}

1. Fälle, in denen direkte Autoxydation möglich ist.

Im folgenden sollen bekannte biologische Oxydationsreaktionen von den erörterten Gesichtspunkten aus besprochen

1) Hier sei auf die bekannte Cannizzarosche Reaktion hingewiesen, bei der sich gleichzeitig eine Oxydation und eine Reduktion abspielt; vgl. auch E. u. W., S. 142 ; ferner Martinand, C. R., 1909, Sitzungsber. vom 18. Januar.

8) Vgl. z. B. Oppenheimers Handbuch der physiol. Chemie, Bd. II, 1. Hälfte, S. 148. 
werden. ${ }^{1}$ ) Nach diesen erscheinen offenbar die direkten Autoxydationsprozesse als die einfachsten. Es sind nun eine ganze Reihe von biochemischen Vorgängen bekannt, die als direkte Autoxydation verlaufen können. Zunächst mögen also solche angeführt werden.

Hydroxylamin stellt bekanntlich eine sehr additionsfähige Verbindung vor; im Organismus wird es unter Sauerstoffaufnahme in salpetrige Säure umgewandelt. ${ }^{2}$ )

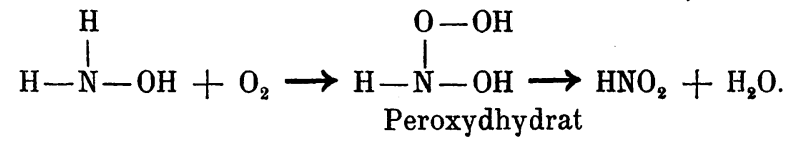

In diesem wie in anderen Fällen entstehen höchst wahrscheinlich erst Additionsprodukte des Autoxydators und Sauerstoffs, die sich allmählich im Sinne der Stufenregel in stabilere Verbindungen umlagern. ${ }^{2}$ )

Phenylhydroxylamin setzt sich im Organismus nach Lewin ${ }^{3}$ ) in Azoxybenzol um. Wie Bamberger ${ }^{4}$ ) gefunden hat, bildet sich bei 60-70 stündigem Durchleiten von Sauerstoff durch eine trockene Lösung von Phenylhydroxylamin in Benzol (neben unerheblichen Mengen Peroxyd) in der Hauptsache Azoxybenzol.

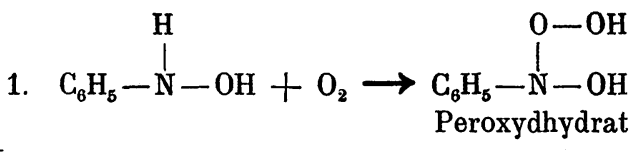

1) Natürlich kann im folgenden nur auf chemisch wohl definierte Prozesse eingegangen werden, biologisch hochinteressante Vorgänge wie die Beeinflussung der Befruchtung durch 0 (J. Loeb, Biochem. Zeitschr., Bd. I, S. 183 (1906); Bd. II, S. 321 (1906); Pflügers Arch., Bd. CXIII, S. 487 (1906); Bd. CXVIII, S. 30 (1907) u. a. a. 0.; W. Ostwald, Biochem. Zeitschr., Bd. VI, S. 409 (1907); 0. Warburg, Diese Zeitschrift, Bd. LVII, S. 1 (1908); vgl. auch Jakoby, Diese Zeitschrift, Bd. XXXIII, S. 128 (1901) u. a.); die Glykolyse (vgl. z. B. Stoklasa, Diese Zeitschrift, Bd. L, S. 303, 1907) und ähnliches können zurzeit nicht erörtert werden. Auch auf die Besprechung der Bedeutung der Autoxydation bei der Antisepsis (vgl. Berthelot, C. R., Bd. CXXXVII, S. 953, 1904) u. dgl. muß verzichtet werden.

2) Binz, Virchows Archiv, Bd. CXIII, S. 1 (1888).

$\left.{ }^{3}\right)$ Arch. f. exp. Path., Bd. XXXV, S. 401 (1895).

$\left.{ }^{4}\right)$ B. B., Bd. XXXIII, S. 113 (1900). Vgl. E. u. W., S. 66. 
2.

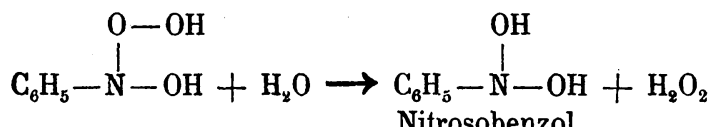

3.

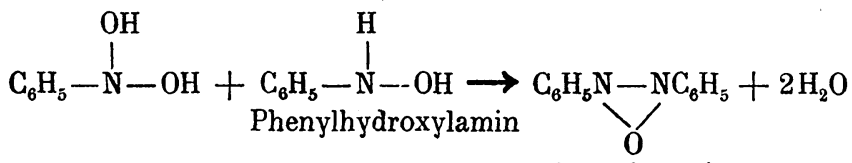

Azoxybenzol.

Aldehyde. Formaldehyd wird ebenso wie seine Natriumbisulfitverbindung im Organismus zu Ameisensäure oxydiert. ${ }^{1}$ ) Acetaldehyd und Paraldehyd werden offenbar zuerst zu Essigsäure oxydiert, die dann zum Teil verbrannt wird. ${ }^{2}$ ) Acetaldol wird in Acetessigsäure umgewandelt. ${ }^{3}$ ) Benzaldehyd wird $z u$ Benzoesäure $^{4}$ ) oder Benzamid. ${ }^{5}$ ) Die drei Nitrobenzaldehyde zeigen an Hunde verfüttert ein verschiedenes Verhalten. Die o-Verbindung, die allerdings $\mathrm{zu} \mathrm{90 \%} \mathrm{verschwindet,} \mathrm{wird} \mathrm{zur}$ Säure, die m-Verbindung unter teilweiser Reduktion zu m-Amidobenzoesäure, der p-Nitrobenzaldehyd zur p-Nitrobenzoesäure und p-Amidobenzoesäure ${ }^{6}$ ) dabei treten noch Unterschiede in der Paarung mit anderen Stoffen auf, die p- und m-Verbindungen erscheinen als Hippursäuren, die o-Säure ungepaart im Harn. Salicylaldehyd wird zur Säure oxydiert ; ${ }^{7}$ ) ebenso Gentisin-

1) Pohl, Arch. f. exp. Path., Bd. XXXI, S. 292 (1893); Kluber, Münchener Med. Wochenschr., Bd. XXXVII, S. 1416 (1900); Gerlach, das., Bd. XLIX, S. 1503 (1902).

2) Cohn, Diese Zeitschrift, Bd. XVII, S. 274 (1892); Reitzenstein, Diss. Würzburg (1894).

s) Friedmann, Hofmeisters Beitr., Bd. XI, S. 202 (1908).

$\left.{ }^{4}\right)$ Wöhler und Frerichs, Liebigs Ann., Bd. LXV, S. 335 (1848).

5) R. Cohn, Diese Zeitschrift, Bd. XIV, S. 203 (1889).

6) Si eber und Smirnow, Monatsh. f. Chem., Bd.VIII, S. 88 (1887). Gohn, Diese Zeitschrift, Bd. XVII, S. 274 (1892); Bd. XVIII, S. 133 (1893).

$\left.{ }^{7}\right)$ Schmiedeberg, Arch. f. exp. Path., Bd.XIV, S. 288, 379 (1881).Jaquet, das., Bd. XXIX, S. 386 (1892). - Salkowski, Zentralbl. für med. Wiss., Bd. XXXII, S. 913 (1895); Virchows Arch., Bd. CXLVII, S. 1 (1897). - Medwedew, Pflügers Arch., Bd. LXV, S. 249 (1896); Bd. LXXIV, S. 193 (1899); Bd. LXXXI, S. 540 (1900); Bd. CIII, S. 403 (1904). - Jakoby, Virchows Arch., Bd. CLVII, S. 235 (1899); Diese Zeitschrift, Bd. XXX, S. 135 (1900). - R. O. Herzog, das., Bd. XXXVII, S. 396 (1903). 
aldehyd, $\left.{ }^{1}\right)$ Protokatechualdehyd, ${ }^{2}$ ) Vanillin, ${ }^{3}$ ) Isovanillin, $\left.{ }^{2}\right)$ Methylvanillin, ${ }^{2}$ ) Piperonal, ${ }^{4}$ ) Furfurol, ${ }^{5}$ ) Thiophenaldehyd. ${ }^{6}$ ) Von Aldehyden ist bekannt, daß sich viele wahrscheinlich unter vorübergehender Peroxydbildung leicht oxydieren : ${ }^{7}$ )

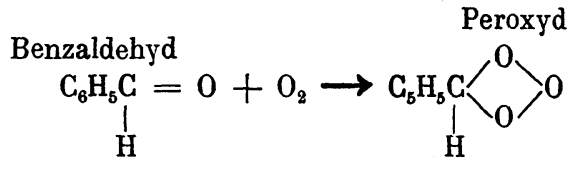

(Aus dem Peroxyd bildet sich mit dem überschüssigen Aldehydd Benzoesäure.)

Phosphor wird im tierischen Organismus meist schwer oxydiert. ${ }^{8}$ ) Es wäre möglich, daß die Langsamkeit, mit der der Phosphor angegriffen wird, daher stammt, daß er in den Lipoiden gelöst und so dem lebhaftesten Oxydationsbetrieb im Organismus entzogen wird. Anderseits war aber gerade die schwere Angreif barkeit des Phosphors im Gegensatz z. B. zu Benzylalkohol und Oxybenzaldehyd für Schmiedeberg ${ }^{9}$ ) der Grund zu der Annahme, «daß das Gewebe bei der Vermittlung

1) Likhatscheff, Diese Zeitschrift, Bd. XXI, S. 422 (1895).

8) Marfori, Chem. Zentralbl., Bd. II, S. 155 (1890).

8) Preusse, Diese Zeitschrift, Bd. IV, S. 209 (1880). - Marfori, 1. c. - Kotake, Chem. Zentralbl., Bd. II, S. 690 (1905).

4) Heffter, Arch. f. exp. Path., Bd. XXXV, S. 349 (1895).

5) Jaffé und Cohn, B. B., Bd. XX, S. 2311 (1887); Bd. XXI, S. 3461 (1888).

$\left.{ }^{6}\right)$ Cohn, Diese Zeitschrift, Bd. XVII, S. 274 (1892).

7) E. und W., S. 87. - Bach, Monit. Scient., S. 479 (1897).

8) Vgl. E. und W., S. 61. -- Kobert, Lehrb. der Intoxikationen, 1904, Bd. II, S. 288. - Arsentrioxyd soll nach Binz und Schulz, Arch. f. exp. Pathol., Bd. XI, S. 200 (1879), Bd. XIV, S. 235 (1881), zu Arsenpentoxyd umgewandelt werden. M. Tonigutti, Chem. Zentralbl., Bd. I, S. 969 (1908) hat aber gezeigt, daß es durch den Organismus nicht verändert wird. Vielleicht sind aber gewisse Lichterscheinungen, die von Organismen hervorgerufen werden, hier zu erwähnen. So hat Beijerinck, Arch. Néerl., Bd. XXIII, S. 116 (1889); Bot. Zg., Bd. XLVIII, S. 744 (1890); Koninkl. Akad. van Wetensch., Amsterdam (1901), S. 45, gezeigt, daß Photobakterien ein empfindlicheres Reagens für Spuren freien Sauerstoffes abgeben als Indigweiß, und sie auch zum Nachweis der Chlorophyllassimilation der Algen benutzt. Vgl. auch Molis ch, \&Leuchtende Pflanzen ?.

9) l. c.

Hoppe-Seyler's Zeitschrift f. physiol. Chemie. LIX. 
der Oxydation nicht auf den Sauerstoff, sondern auf die oxydierbaren Substanzen einwirkt, indem es sie jenem zugänglicher macht».

In Wirklichkeit liegt die Sache wohl so, daß auch der direkten Autoxydation fähige, körperfremde Stoffe im Organismus zumeist eher indirekt autoxydiert werden. ${ }^{1}$ ) Hauptsächlich wohl aus dem Grunde, weil, wie bereits erwähnt, die indirekten Autoxydationen schneller verlaufen, und weil notwendig sehr reaktionsfähige direkte Autoxydatoren unter den Agenzien der Organismen sich finden müssen, in deren Gegenwart andere Autoxydatoren leicht zum Acceptor werden. Dazu kommt, daß möglicherweise der Oxydation eine Art von Bindung an die "Gewebe» vorausgeht, wie auch Schmiedeberg und Pfeffer andeuten. So werden die behandelten Oxydationsreaktionen zumeist nicht Autoxydationsvorgänge, sondern Oxydationen vorstellen. Die Stoffe im Organismus, deren wesentliche Rolle für das Leben darin besteht, daß sie autoxydabel sind, und zu denen wohl auch die Oxygenasen gehören, sind wohl der Hauptsache nach noch völlig unbekannt. Naturgemäß ist es unter diesen Umständen noch schwerer als in den meistens einfacheren, rein chemischen Fällen die Frage zu beantworten, welche von allen möglichen Reaktionen stattfindet. Ganz allgemein läßt sich, wie ja bereits erwähnt, höchstens sagen, daß die Geschwindigkeit der Reaktion zumeist ausschlaggebend sein wird; wovon aber wiederum die Geschwindigkeit abhängt, ist eine Frage, für deren Beantwortung die Grundlagen heute fast durchwegs fehlen.

2. Fälle, in denen indirekte Autoxydation möglich ist.

Als Schema der indirekten Autoxydation diente die Gleichung:

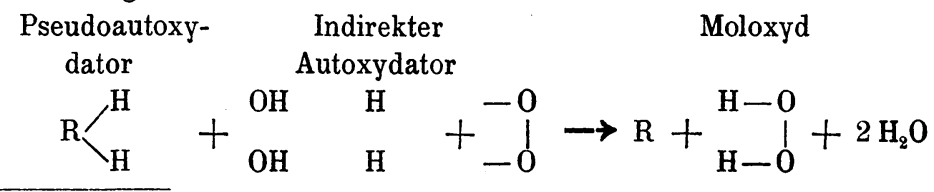

1) Z. B. hat auch eine eingehendere Untersuchung im hiesigen Institut durch Herrn Speithel ergeben, daß keineswegs alle der oben angeführten Aldehyde direkt autoxydabel sind. 
Es wurde auch bereits erwähnt, daß als Pseudoautoxydatoren eine ganze Reihe von chemischen Verbindungen auftreten, die leicht Wasserstoff abdissoziieren. $\mathrm{R}$ können auch zwei gleiche oder verschiedene Verbindungen vorstellen, die erst durch die Reaktion verkuppelt werden. Wesentlich für das weitere Bild der Oxydation ist natürlich, ob $\mathrm{R}$ beständig oder weiterhin leicht oxydabel ist; dann schreitet die Reaktion weiter fort, wie bei den Phenolen und Aminen, während die Reste der Leukobasen, die Farbstoffe meist widerstandsfähiger sind. Man beobachtet hier häufig die bekannte Erscheinung, daß das H-Atom in p-Stellung besonders labil ist und leicht abdissoziiert. Die meisten dieser Reaktionen werden durch alkalische Reaktionen katalytisch beschleunigt. ${ }^{1}$ ) Als Schemata für die indirekten Autoxydationen von Leukobasen sei die von Phenolphthalin zu Phenolphthalein und die Indophenolbildung ${ }^{2}$ ) ausgeführt, im übrigen sei auf die Arbeit von Engler und Weißberg verwiesen.

Phenolphthalin ${ }^{3}$ )

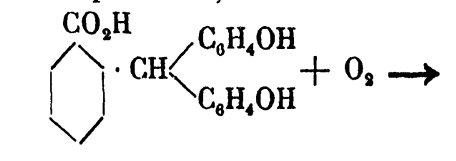

in alkalischer Lösung: $\mathrm{H}_{2} \mathrm{O}_{\mathbf{2}}+$<smiles>O=C1CCC2C3=C1C2=C(C1CCCCC1)C(=O)CC3</smiles>

saurer

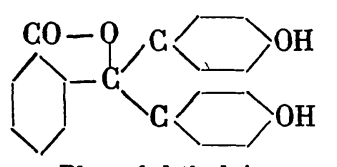

Phenolphthalein. (1904).

1) Vgl. E. und W., S. 184; Friedenthal, Festschr. f. Salkowski

2) Röhmann und Spitzer, B. B., Bd. XXVIII, S. 567 (1895); Über die Konstitution s. Möhlau, das., Bd. XVI, S. 2843. Vgl. D. R. P., Kl. $12-$ q-A. 12284, 122885, Verfahren zur Darstellung von Leukokörpern der Indophenolreihe.

3) Um von der allgemeinen Gewohnheit nicht abzuweichen, sind hier die Formeln abgekürzt geschrieben, d. h. ohne die Mitwirkung des Wassers zum Ausdruck zu bringen; s. S. 332. 


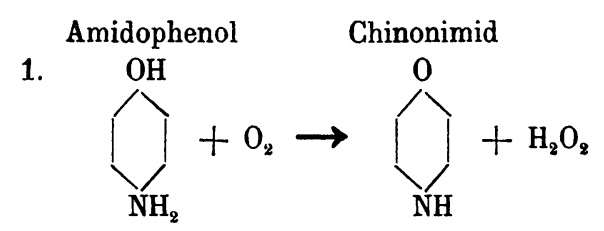

2.

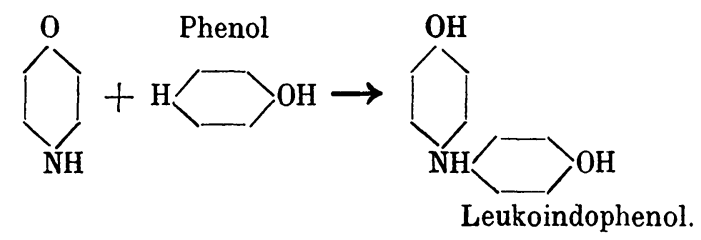

3.

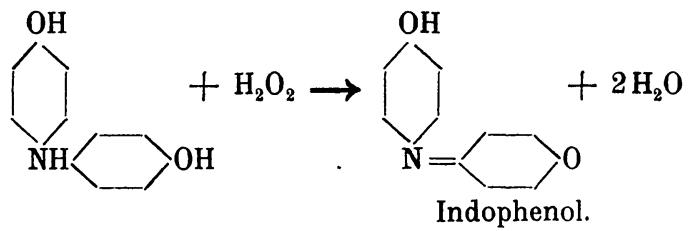

Ein schönes hier anzuführendes Beispiel ist Cystein, das unter Autoxydation bekanntlich sehr leicht in Cystin übergeht, ohne Zweifel ein Analogon der von Engler und Broniatowski ${ }^{1}$ ) näher untersuchten Autoxydation des Thiophenols zu Phenylbisulfid.

Ferner gehört die Jodidoxydation hierher.

Als indirekte Autoxydation wird man wohl auch die von Schmiedeberg ${ }^{2}$ ) gefundene Umwandlung von Benzylalkohol in alkalischer Lösung betrachten müssen; wie R. O. Herzog und Polotzky gefunden haben, bildet sich beim Behandeln des Benzylalkohols mit wässerigem Alkali oder Pottasche in der Kälte und in der Hitze zunächst Benzaldehyd, wenn man Sauerstoff durch die Lösung resp. Emulsion streichen läßt. ${ }^{3}$ ) Welche Rolle das Alkali bei dieser Art Vorgänge spielt, ist bekanntlich noch nicht sichergestellt; möglich ist, daß die Dissoziationsverhältnisse in Betracht kommen, wie Manchot 4 ) meint; es könnten aber auch die Alkalimetallionen autoxydabel sein. ${ }^{5}$ )

1) B. B., Bd. XXXVII, S. 3274 (1904).

2) Arch. f. exp. Path., Bd. XIV, 1. c.

$\left.{ }^{3}\right)$ Es werden weitere Versuche nach dieser Richtung angestellt.

4) Habilitationsschrift, 1900.

5) E. und W., S. 141. - Vgl. auch diese Abh. S. 340. 
$\mathrm{Zu}$ den indirekt autoxydabeln Stoffen gehören die meisten sogenannten Oxydasereagenzien, die später noch zu erwähnen sind. Hier mögen nur noch die Umwandlungen von Alkoholen, Phenolen und Aminen im Organismus angeführt werden, die sämtlich durch alkalische Reaktion begünstigt, als indirekte Autoxydationen zu verlaufen vermögen. Daß die chemische Konstitution für die Leichtigkeit, mit der sich die Reaktionen abspielen usw., maßgebend ist, darf wohl nicht besonders erörtert werden. Da außerdem allgemein gültige Regeln nach dieser Richtung bislang fast vollständig fehlen, böte wohl auch die detaillierte Besprechung des Bekannten nichts Wesentliches.

Phenole. Phenol ${ }^{1}$ ) wird in Hydrochinon und Brenzkatechin umgewandelt. Oxydasen reagieren mit Guajakol, ${ }^{2}$ ) im Organismus geht es in Pyrogallol und Oxyhydrochinonderivate über. ${ }^{3}$ ) Resorcin, ${ }^{4}$ ) Kresole ${ }^{5}$ ) werden oxydiert, Carbonylo-Aminophenol wird zu Carbonyl-0-0xyaminophenol, ${ }^{6}$ ) Phenetol ${ }^{7}$ ) wird zum Äthyläther des Hydrochinons.

Mitunter greift bei den Oxybenzolen, Oxynaphtolen, also der Sauerstoff den Kernwasserstoff an. ${ }^{8}$ ) Einfacher ist aber der Fall, daß der Hydroxylwasserstoff oxydiert oder auch autoxydiert wird. Ein Beispiel letzterer Art ist die von Manchot ${ }^{9}$ ) untersuchte Autoxydation von Anthrahydrochinon zu Anthrachinon:

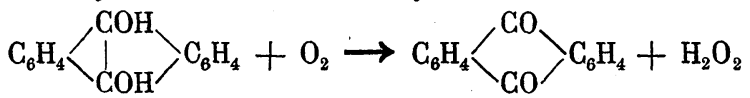

Natürlich ist auch noch möglich, daß beides eintritt, die

1) Tauber, Diese Zeitschrift, Bd. II, S. 366 (1878). - Schaffer, Journ. f. prakt. Chem., Bd. XVIII, S. 282 (1878). - Baumann und Preusse, Diese Zeitschrift, Bd. III, S. 156 (1879).

2) G. R., Bd. CXLl, S. 891 (1905).

$\left.{ }^{3}\right)$ Eschle, Z. f. klin. Med., Bd. XXIX, S. 197 (1896).

4) Kymmyser und Stockvis, J. B. f. Tierchem., Bd. XIII, S. 213 (1883); das., Bd. XIX, S. 462 (1889).

5) Biochem. Z., Bd. I, S. 399 (1906).

$\left.{ }^{6}\right)$ Gressly und Nencki, Monatsh. f. Chem., Bd. XI, S. 253 (1890).

7) Kossel, Diese Zeitschrift, Bd. IV, S. 296 (1880). - Kühling, Diss. Berlin, 1887. - Lehmann, Diese Zeitschrift, Bd. XIII, S. 181 (1888).

8) E. und W., S. 128.

9) Liebigs Ann., Bd. CGGXIV, S. 179 (1901). 
Oxydation des Kernwasserstoffs und des Hydroxylwasserstoffs, dann treten nebeneinander Chinone und Hydrochinone auf.

Amine. Anilin wird $\mathrm{zu}$ p-Aminophenol, ${ }^{1}$ ) ebenso (nach vorausgegangener Hydrolyse) Formanilid, Azetanilid desgleichen, zum Teil aber auch zur o-Oxyphenylcarbaminsäure, ${ }^{2}$ ) Benzidin zu Diaminodioxydiphenyl, ${ }^{3}$ ) Phenylurethan zu p-Oxyphenylurethan, ${ }^{4}$ ) Carbazol zu Oxycarbazol, 5) Dimethylanilin zu Oxydimethylanilin,,$^{6}$ ) Diphenylamin zu p-Oxydiphenyl ${ }^{7}$ ) oxydiert. Zu erwähnen wären hier noch Tyrosin, Phenylalanin und die von ihnen abstammenden Peptide.

Alkohole. Methylalkohol geht im Organismus in Ameisensäure über. ${ }^{8}$ ) Weiter wäre hier an die Oxydation der Alkohole durch Gärungserreger zu erinnern. ${ }^{9}$ ) Äthylenglykol liefert im Organismus Glykolsäure ${ }^{10}$ ) resp. Oxalsäure; auch Propylenglykol scheint oxydiert zu werden. ${ }^{11}$ ) Aus Acetaldol wird Acetessigsäure und Aceton, ${ }^{12}$ ) aus $\beta$-Oxybuttersäure ${ }^{13}$ ) ebenfalls. d-glu-

1) Külz, Pflügers Arch., Bd. XXX, S. 484 (1883). - Schmiedeberg, Arch. f. exp. Path., Bd. XIV, S. 288 (1881). - Engelhardt, Diss. Dorpat, 1888.

2) Diese Zeitschrift, Bd. XXII, S. 327 (1896). - Cahn und Hepp, Berl. Klin. Wochenschr., 1887, Nr. 1 u. 2. - F. Müller, Ther. Monatsh., Bd. II, S. 355 (1888). - Della Cella, Journ. de pharm. et de chim. (5), Bd. XV, S. 462 (1887). - Jaffé und Hilbert, Diese Zeitschrift, Bd. XII, S. 295 (1888). - Mörner, das., Bd. XIII, S. 12 (1888).

3) Arch. f. exp. Path., Bd. LVIII, S. 167 (1908).

4) J. B. f. Tierchem., Bd. XXI, S. 46 (1891).

$\left.{ }^{5}\right)$ Klingenberg, Diss. Rostock, 1891. - Fühner, Arch. f. exp. Pathol., Bd. LI, S. 391 (1904).

6) Diese Zeitschrift, Bd. I, S. 244 (1877/78).

${ }^{7}$ ) Diss. Rostock, 1891.

$\left.{ }^{8}\right)$ Pohl, Arch. f. exp. Path., Bd. XXXI, S. 281 (1893). - Es sei hier erwähnt, daß Methylaminchlorhydrat nach Pohl, 1. c., ebenfalls Ameisensäure liefert.

9) Siehe S. 365 .

10) Mayer, Diese Zeitschrift, Bd. XXXVIII, S. 135 (1903). - Pohl, Arch. f. exp. Path., Bd. XXXVII, S. 413 (1896).

11) Neubauer, Arch. f. exp. Path., Bd. XLVI, S. 133 (1901).

$\left.{ }^{12}\right)$ Friedmann, Hofmeisters Beitr., Bd. XI, S. 202 (1908).

13) Araki, Diese Zeitschrift, Bd. XVIII, S. 1 (1893). - Waldvogel, Zentralbl. f. inn. Med., Bd. XIX, S. 845 (1898). - Zeehüisen, J. B. f. Tierchem., Bd. XXIX, S. 825 (1899). 
konsaures Natrium liefert d-Zuckersäure. ${ }^{1}$ ) Die Umwandlung des Benzylalkohols in Benzoesäure wurde bereits erwähnt, ihr entspricht vollständig die von Saligenin in Salicylsäure, ${ }^{2}$ ) von Furfuralkohol in Brenzschleimsäure. ${ }^{3}$ )

3. Stoffe, die als Acceptoren wirken können.

Weitaus die meisten Oxydationsreaktionen spielen sich, wie oben ausgeführt wurde, an Stoffen ab, die als Acceptoren wirken. Es ist auch bereits auseinandergesetzt worden, daß sowohl direkte wie Pseudoautoxydatoren zu Acceptoren werden können, d. h. also den Sauerstoff nicht direkt oder durch Vermittlung der Wasserstoffionen aufnehmen, sondern daß sie, ebenso wie viele von den ersteren, als Selbstacceptoren ihren Superoxyden gegenüber funktionieren, auch von fremden Peroxyden oder anderen reduzierbaren Verbindungen Sauerstoff aufzunehmen vermögen. Auf die induzierten und katalytischen Vorgänge, die solche Prozesse verursachen können, wird im folgenden Abschnitt eingegangen werden. Natürlich gilt auch hier, wie bei der Autoxydation, daß die Angreifbarkeit des Oxydationsproduktes entscheidet, ob es als solches auftritt, oder weiter verändert wird.

Über die chemische Natur der Stoffe, die als Acceptoren zu funktionieren vermögen, läßt sich nur sagen, daß sie wie Autoxydatoren und Pseudoautoxydatoren mehr oder weniger ungesättigte und solche Verbindungen darstellen, die leicht dissoziationsfähig sind. (Dabei kann sowohl saure wie alkalische Reaktion begünstigenden Einfluß nehmen.) Allerdings lassen sich im allgemeinen genauere Kennzeichen für die Fähigkeit eines Stoffes, als Acceptor aufzutreten, nicht geben, zumal vielleicht mehr noch die Reaktionen von weniger additionsfähigen, wenigerungesättigten, weniger dissoziierenden Stoffen von fremden Einflüssen abhängen werden als die der eminent reaktionsfähigen. Es spielen also die speziellen Einflüsse, vor allem aber die Natur des Oxydationsmittels eine sehr große Rolle, die man im Prinzip

1) Mayer, B. B., Bd. XXXIV, S. 492 (1901).

$\left.{ }^{2}\right)$ Nencki, Arch. f. Anat. u. Physiol., Physiol. Abt., 1870, S. 399.

s) Erdmann, Arch. f. exp. Path., Bd. XLVIII, S. 255 (1902). 
nicht anders erklären kann, als daß es sich um bestimmte Verbindungen (Additionsprodukte aus dem Oxydationsmittel oder einem anderen beeinflussenden Stoff und dem Acceptor) oder um eine Verschiebung der Dissoziationsverhältnisse handelt. Darum wird auch das allgemein zu Erwähnende wohl besser in dem Abschnitt über katalytische und induzierte Reaktionen besprochen.

Es hat somit wohl auch nicht viel Zweck, solange eine genaue Erkenntnis der Agenzien und Zwischenprodukte fehlt, die sich z. B. mit normalen Bestandteilen des Organismus als einem Paarling bilden können, hier auf gewisse, vielleicht für die Zukunft viel versprechende Regelmäßigkeiten einzugehen, wie sie durch die Regeln von Schotten ${ }^{1}$ ) und Knoop ${ }^{2}$ ) ausgedrückt werden, aus den Arbeiten von Embden ${ }^{3}$ ) und seinen Mitarbeitern, E. Friedmann, $\left.{ }^{4}\right)$ Dakin $^{5}$ ) u. a. hervorzugehen scheinen. Das Wesen solcher Gesetzmäßigkeiten soll im folgenden seine Deutung finden, soweit dies eben möglich ist.

\section{Katalytische und induzierte Reaktionen.}

\section{Geschichtliches.}

E. Mitcherlich ${ }^{6}$ ) hat solche chemische Vorgänge als "Kontaktwirkungen» bezeichnet, bei denen die Anwesenheit eines Stoffes für das Zustandekommen einer Reaktion nötig ist, ohne daß dieser selbst merkbar an der Reaktion teilnimmt, ohne daß er also in der chemischen Reaktionsgleichung angeführt wird. ${ }^{7}$ ) J. Berzelius ${ }^{8}$ ) verwandte bekanntlich für die von Mitcherlich bei seinen Studien über die Bildung des Äthers untersuchten Erscheinungen das Wort "Katalyse». Sie besteht darin, «daß

1) Diese Zeitschrift, Bd. VII, S. 23 (1882); Bd. VIII, S. 65 (1883/84). Vgl. auch Nencki u. Giacosa, Diese Zeitschr., Bd. IV, S. 337 (1880).

2) Habilitationsschr., 1904.

3) Hofmeisters Beitr., Bd. VIII, S. 129; Bd. XI, S. 318, 323, 327, 332 ; Biochem. Zeitschr., Bd. XIII, S. 262 (1908).

4) Hofmeisters Beitr., Bd. XI (1908).

s) Journ. of Biolog. Chem., Bd. I-V.

6) Pogg. Ann., Bd. XXXI. S. 273 (1834).

7) Siehe Bodenstein, Z. Elektrochem., Bd. IX, S. 735 (1903).

s) J. B., Bd. XV, S. 237 (1836). 
Körper durch ihre bloße Gegenwart und nicht durch ihre Verwandtschaft die bei dieser Temperatur schlummernden Verwandtschaften zu erwecken vermögen.» ${ }^{1}$ ) Er führt als hierher gehörige Fälle außer der Ätherbildung durch Schwefelsäure unter andern an die Zuckerbildung aus Stärke durch Schwefelsäure, sowie durch Diastase und besonders noch die Zersetzung von Wasserstoffsuperoxyd durch Platin, Silber oder Faserstoff, ferner die ihr so sehr gleichende «Umwandlung des Zuckers in Kohlensäure und Alkohol, wie sie bei der Gärung durch den Einfluß eines unlöslichen Körpers stattfindet, den wir unter dem Namen Ferment kennen und der, wie wohl mit geringerer Wirksamkeit, durch tierischen Faserstoff koaguliertes Pflanzeneiweiß, Käse und ähnliche Substanzen ersetzt werden kann.» ${ }^{2}$ ) Berzelius hat also bereits die Fermente - im heutigen Sinne - zu den Katalysatoren gezählt. ${ }^{3}$ )

Wie man weiß, hat sich Liebig ${ }^{4}$ ) gegen die «katalytische Kraft », von der Berzelius sprach, gewendet und dafür die Ursache von "Verwesung, Fäulnis, Gärung und Vermoderung * in der Fähigkeit gesehen, welche ein in Zersetzung oder Verbindung, d. h. in einer chemischen Aktion begriffener Körper besitzt, in einem andern ihn berührenden Körper dieselbe Tätigkeit hervorzurufen oder ihn fähig zu machen, dieselbe Veränderung zu erleiden, die er selbst erfährt». ${ }^{5}$ ) Es ist offensichtlich, daß Liebig zu seiner Anschauung durch Verallgemeinerung der Beobachtungen gerade an Gärungs- und Fäulnisprozessen gelangt ist. ${ }^{6}$ ) Wahrscheinlich wollte er, wie auch Skrabal ${ }^{7}$ ) meint, ursprünglich von der Erfahrungstatsache ausgehend, daß die Gärungs-‘Fermente» sich bei ihrer Wirkung verändern, zunächst eine Klassifikation der Vorgänge geben; der Schluß

1) J. B., Bd. XV, S. 273 (1836).

2) Dass., S. 240.

s) Vgl. auch sein Lehrb., III. A. (1835-41), Bd. VI, S. 19.

4) Pogg. Ann., Bd. XLVIII, S. 106 (1839).

5) Dass., S. 118.

6) Vgl. bes. die org. Chem. in ihrer Anw. auf Agrikulturchemie und Physiologie. Die Literatur ges. bei Volhard, J. v. Liebig, 1909, Bd. II, S. 75, Anm. 1.

7) Die induc. Reaktionen, Samml. chem. und tech. Vortr. 1908. 
der genannten Abhandlung ${ }^{1}$ ) spricht dafür. Später ist ihm aber zumal bei wachsender Mißstimmung gegenüber Berzelius jedenfalls wesentlich seine Theorie am Herzen gelegen. Dabei berücksichtigt er anfangs, wie bekannt, auch den von CiagniardLatour, $\left.{ }^{2}\right)$ Schwann ${ }^{3}$ ) und Kützing ${ }^{4}$ ) erbrachten Nachweis des Lebens der Hefezellen nicht; erscheint doch 1828 in den Annalen ${ }^{5}$ ) die bekannte Satire Wöhlers, zu der Liebig «noch einige schlechte Späße hinzugemacht hat». ${ }^{6}$ ) Er glaubt damals so wenig, wie die meisten anderen Chemiker, an die Zuverlässigkeit der botanischen Beobachtungen. Später ${ }^{7}$ ) ersieht er in ihnen nur eine nicht wesentliche Komplikation, was ja auch richtig ist. $\left.{ }^{8}\right)^{*}$ )
1) S. 144 .
2) Ann. d. chim. et phys. (2), Bd. LVIII, S. 216 (1836).
$\left.{ }^{3}\right)$ Pogg. Ann., Bd. CXVII, S. 184 (1837).
4) Journ. f. pr. Chem., Bd. XI, S. 387 (1837).
5) Ann., Bd. 29, S. 100.
6) Briefwechsel zwischen Liebig und F. Wöhler, herausg. von A. W. Hofmann, 1888, L's. Brief vom 18. Nov. 1838, vgl. auch W's. Brief vom 2. März 1839.

7) Handwörterbuch der Chemie 1848.

$\left.{ }^{8}\right)$ Vgl. die Darstellung in Volhard, Liebig, Bd. II, S. 72, Dasselbe findet sich auch bei Schönbein (Zeitschr. f. Biol., Bd. I, S. 280, 1865): $\varangle \mathrm{Da}$ es Fermente gibt, die trotz ihrer organischen Natur doch nicht organisiert sind, wie z. B. das in den Mandeln oder dem schwarzen Senf enthaltene Emulsin und Myrosin, so bin ich geneigt zu glauben, daß auch die durch bestimmte Tierchen oder Pflänzchen verursachten Gärungen mit der Organisation dieser Gebilde als solcher nichts zu tun haben und nur die eigentümliche stoffliche Beschaffenheit des Materials, aus welchem die Organismen bestehen, es sei, durch welche ächte Gärungserscheinungen hervorgerufen werden.»

*) In J. Mulders bekanntem «Versuch einer allgemeinen physiologischen Chemies aus jener Zeit (Braunschw. 1844-51) findet sich die Unterscheidung dreier Arten chemischer Wirkungen ähnlich wie bei Liebig (S. 32).

1. -Diejenige, welche von einem Stoff ausgeht, ohne ihn selbst zu affizieren, sondern welche bloß auf andere Körper übertragen wird (Katalyse).

2. Diejenige, welche von einem Stoff auf einen anderen übergeht, wobei auch der erstere sich zersetzt, ohne indes den neuen Produkten einen seiner Bestandteile zu leihen (Fermentation). 
Nicht viel später als in dieser ersten Zeit der Diskussion über Katalyse beginnen die physiologisch-chemischen Unter-

3. Diejenige, welche auf die Stoffe, von denen sie ausgeht, zurückwirkt, wo beide an der Zersetzung teilnehmen und gemeinschaftliche Produkte liefern (gewöhnliche chemische Wirkung).»

Trotz mancher erheblicher Mißverständnisse in der weiteren Behandlung, neben denen sich auch wieder logisch berechtigte Einwände gegen Liebig finden, liegt hier eine klare Systematik vor. Die ganze Auffassung ist freilich durch Störungen getrübt, welche den Versuchen jener Zeit durch Infektionen mit Mikroorganismen anhaften. Dies zeigen auch deutlich die entsprechenden Stellen anderer physiologischer Lehrbücher dieser Jahre. (Vgl. z. B. C. Ludwig, I. A. 1852, Bd. I, S. 41-43.)

R. F. Marchand spricht in seinem Lehrbuch der physiologischen Chemie (Berlin 1844) völlig im Sinne von Mitcherlich und Berzelius über Kontakterscheinungen und katalytische Kraft; auch der Warnung Berzelius' schließt er sich an: "Geht man soweit zu glauben, durch dieses Wort eine Erklärung gegeben zu haben, so hat man keinen Fortschritt, sondern einen Rückschritt in der Wissenschaft gemacht.» Da der ganze Abschnitt zu den klarsten und einwandfreiesten Darstellungen der Zeit gehört, mag hier reproduziert sein, was sich speziell auf die Enzyme bezieht (S. 57-58). «Einige tierische Stoffe besitzen namentlich das Vermögen, organische Verbindungen aufzuheben und in andere umzuwandeln, gleichfalls nur dadurch, daß sie gegenwärtig sind. Sie selbst erleiden scheinbar keine Veränderung. Der Lab macht durch seine Gegenwart die Milch gerinnen, ohne selbst dadurch modifiziert zu werden; tierischer Schleim, in einer schwachen Säure aufgelöst, besitzt eine außerordentlich starke auflösende Kraft für gewisse organische Verbindungen (Nahrungsmittel, künstliche Verdauung); tierische Schleimhaut verwandelt den Traubenzucker in Milchsäure usw. Alle diese Phänomene gehören scheinbar in dieselbe Klasse, wie die oben angeführten. Wir wissen von ihnen nichts anderes, als daß sie existieren; wir nennen sie daher katalytische. Es ist höchstwahrscheinlich, daß im animalischen und vegetabilischen Organismus dergleichen Erscheinungen sehr häufig auftreten, und daß infolge derselben eine große Anzahl von Veränderungen im Körper vorgeht, welche wir aus dem Grunde nicht nachahmen können, weil uns das Vermögen fehlt, diese Kraft willkürlich hervorzubringen.

Wir sehen diese Art von Tätigkeit namentlich an gewisse tierische Stoffe gefesselt, und wir sind daher imstande, durch eben diese Stoffe (Schleimhäute u. a. m.) Wirkungen hervorzubringen, welche die größte Übereinstimmung mit den im Körper selbst auftretenden zeigen; aber auch nur durch Anwendung dieser Stoffe können wir dies erreichen. Wir dürfen nun annehmen, daß die katalytische Kraft im Körper sehr verbreitet ist, und daß es vorzüglich Zersetzungserscheinungen sind, welche 
suchungen Chr. Fr. Schönbeins ${ }^{1}$ ) über die Sauerstoffwirkung. Es ist außerordentlich interessant, $z u$ sehen, wie er im allgemeinen die Verhältnisse bei katalytischen und - wie wir heute sagen würden - induzierten Vorgängen richtig erfaßt!2) Er erkennt, "daß es eine große Anzahl von Tatsachen gibt, die zugunsten der Annahme" Liebigs sprechen, und glaubt bei gewissen Oxydationsvorgängen an «Übertragung der chemischen

dadurch herbeigeführt werden; diese aber führen alsbald auch die Entstehung neuer Verbindungen herbei; denn es tritt das dafür günstige Moment, der status nascens auf, welcher, wie ich oben zu zeigen suchte, vorzüglich die Bildung der organischen Verbindungen zu bewirken scheint.

Wie sehr diese Kraft dem Organismus eigentümlich ist, sieht man daraus, daß selbst mit dem Erlöschen des Lebens viele Teile des Körpers sie beibehalten und nach dem Tode ebenso fortwirken können, wie im Leben selbst. Daraus sehen wir auch, daß diese Kraft mehr als eine rein chemische und nicht als eine vital chemische $z u$ betrachten sei, oder wenigstens als eine Kombination beider, in der jene bei weitem vorherrscht.»

Die folgende Literatur steht teils unter dem Einfluß Liebigs, teils unter dem Pasteurs (der übrigens selbst die Frage nach dem Chemismus der Fermentwirkung für seine Person nicht beantworten wollte, Ann. Chim. et phys. (3) Bd. LVIII, S. 360, 1860). So spricht sich Kühne in seinem Lehrbuch der physiologischen Chemie 1866, S. 40 gegen die Theorie aus, "nach welcher der Magensaft einer in fortwährender Bewegung und Umwandlung begriffenen Substanz seine Wịkung verdanke, die eben in einer auf das Fibrin mitgeteilten Bewegung bestehen sollte». Übrigens hat Pasteurs Standpunkt viel Unklarheit zur Folge gehabt (man sehe in Brückes Vorl. über Physiologie, Bd. I, 1847, S. 289). Klar spricht sich darüber Hoppe-Seyler aus, Phys. Chemie, Bd. I, S. 114, 115 (1883). Andere Chemiker und Physiologen führen molekular-physikalische Betrachtungen offenbar im Anschluß an Liebigs Gedanken durch, wie Bunsen (Gasometrische Methoden, S. 267), Kekulé (L. Ann., Bd. CVI, S. 141, 1848); Lehrbuch der org. Chemie 1861, Bd. I, S. 142 . Naumann (Grundriß der Thermochemie 1869, S. 117), L. Meyer (Die modernen Theor. d. Chem., Bd. II, A, S, 273), Hüfner (Journ. f. pr. Chem., Bd. X, S. 148 und 385, 1874), Naegeli (Theorie der Gärung, München 1879). Mit Liebig (Chem. Briefe L. A. 1844, S. 186) kann man diese Gedankengänge mit Recht auf Laplace und Berthollet zurückführen.

1) Literatur bei Kahlbaum und Schaer in Kahlbaums Monographie aus Gesch. d. Chem., H. 6, S. 261 (1901). - Vgl. auch Schaer, Zeitschrift f. Biol., Bd. XXXVII, S. 320 (1899).

$\left.{ }^{2}\right)$ Pogg. Ann., Bd. CLXXVI, S. 34 (1857). 
Tätigkeit eines Körpers auf einen andern».1) Anderseits scheint ihm aber gerade bei der alkoholischen Gärung "möglich, daß die Zersetzung des Zuckers zu derjenigen der Hefe nicht in dem Verhältnis von Wirkung zur Ursache stände, letztere Zersetzung nur eine nebenher laufende und nicht eine die Zuckergärung bedingende Erscheinung wäre». ${ }^{2}$ ) Freilich fehlen noch die sicheren Kriterien und die durch das nicht untrügliche "chemische Gefühl» ersetzt werden müssen. Und gerade die eigenen Arbeiten führen zu Fragen, die heute noch nicht völlig gelöst sind.

Die Wirkung von Platin «auf Gemische von Guajaktinktur mitWasserstoffsuperoxyd, ozonisiertem Terpentinöl, ozonisiertem Äther oder dem Erzeugnis der langsamen Verbrennung des Äthers» bezeichnet Schönbein als "chemische Berührungswirkung» ${ }^{3}$ ) und findet sie analog der Wirkung von Blutkörperchen oder Kleber. Ebenso wirken Platin und Blutkörperchen gleichmäßig auf Gemische von Indigo mit Wasserstoffsuperoxyd oder den genannten Stoffen. Er findet die katalytische Wirkung gegenüber Wasserstoffsuperoxyd und Guajaktinktur, sowie gegen ersteres allein in der organischen Natur überaus verbreitet; ${ }^{4}$ ) außer in den genannten Stoffen findet er sie in Diastase, Myrosin, Kleber, Speichel, Hefe, Kalbsmagen (letzteres nur gegen Wasserstoffsuperoxyd wirksam), in den Kartoffelschalen, Auszügen von Samen usw. Die Vorgänge erscheinen den anderen Fermentreaktionen völlig vergleichbar - er nennt «die durch Platin bewerkstelligte Zerlegung des Wasserstoffsuperoxyds das Urbild aller Gärung». ${ }^{5}$ ) Schon 1856 hat $\mathrm{er}^{6}$ ) in Pilzen *eine Sauerstoff erregende und Sauerstoff übertragende Materie» gefunden, die Guajaktinktur bei Gegenwart von Wasserstoffsuperoxyd blau färbt, dieses Vermögen aber durch Erhitzen verliert, ebenso durch Vergiftung mit Blausäure. ?)

1) Journ. f. pr. Chem., Bd. LXXV, S. 109 (1858).

2) 1. c. S. 37 .

s) Kgl. bayer. Akad. d. Wissensch., Bd. II, Kl. VIII, I. Abt.

4) Journ. f. pr. Chem., Bd. LXXXIX, S. 325 (1863); s. auch Zeitschrift f. Biol., Bd. IV, S. 368 (1868).

s) 1. c. S. 335 .

6) Verh. d. Naturf.-Ges. Basel, 3. Heft.

7) Z. f. Biol., Bd. III, S. 140 (1867). 
Aus den.Publikationen Schönbeins geht mit Sicherheit hervor, daß er der Ansicht ist, mit Stoffen völlig vergleichbar der Diastase und anderen Fermenten zu tun zu haben; das zeigen auch die eigenartigen Gedanken, zu denen ihn das stets vergesellschaftete Vorkommen der von ihm entdeckten Fähigkeiten der Extrakte mit denen bereits bekannter Fermentwirkungen veranlaßt. ${ }^{1}$ ) Aber erst 1878 hat M. Traube ${ }^{2}$ ) das Wort Oxydationsferment geprägt, nachdem er 20 Jahre vorher in seiner Theorie der Fermentwirkungen von "Verwesungsfermenten» gesprochen hat. Hoppe-Seyler, der im allgemeinen, wie ja bekannt, Traubes Theorie energisch entgegentrat, drückt sich gerade in dieser Frage äußerst vorsichtig aus. ${ }^{3}$ ) Die Physiologen der sechziger und siebziger Jahre beschäftigen sich vor allem mit den Oxydationsvorgängen im Blut, eventuell auch mit dem Chemismus derselben. ${ }^{4}$ ) Auch bei Schmiedeberg ${ }^{5}$ ) finden sich in der Arbeit «über Oxydation und Synthesen im Tierkörper» keine definitiven Ansichten über die Natur der Vorgänge. Erst neun Jahre später, 1892, spricht sein Schüler Jaquet ${ }^{6}$ ) wieder von der «Deutung, daß die Oxydation im Tierkörper unter dem Einfluß eines Ferments oder Enzyms zustande kommt». ?)

1) Journ. f. pr. Chem., Bd. LXXXIX, S. 337 (1863).

$\left.{ }^{2}\right)$ Ges. Abh., Berlin 1899, S. 385.

$\left.{ }^{3}\right)$ Phys. Chem., 1881, 1. Teil, S. 126 u. ff.

4) Siehe Claude Bernard und seine Schüler in tier. Wärme. A. Schmidt, Arch. f. pathol. Anat. u. Physiol., Bd. XLII, S. 249. Kühne u. Scholz, Arch. f. pathol. Anat. u. Physiol., Bd. XXXIII, S. 96 (1865). - Lewisson, das., Bd. XXXVI, S. 15 (1866). - Pokrowsky, das., Bd. XXXVI, S. 482 (1866). - C. Ludwig, Sitzungsber. d. math.phys. Kl. d. Kgl. sächs. Akad. d. Wissensch., Bd. XX, S. 12 (1869). E. Pflüger, Arch. der ges. Physiol., Bd. VI, S. 43 (1872); Bd. X, S. 251 (1875); s. bes. S. 300 u. ff. - Krukenberg, Stud. 1880. - P. Ehrlich, -Das Sauerstoff bedürfnis des Organismus *, Berlin 1885, u. a. - Vgl. auch in Hermanns Handbuch der Physiol. 2. Teil, S. 93 (1882).

5) Arch. f. exp. Pathol., Bd. XIV, S. 388 (1881). - Vgl. auch Wurster, B. B., Bd. XXI, S. 1526 (1888).

6) Arch. f. exp. Path., Bd. XXIX, S. 285.

$\left.{ }^{7}\right)$ Neuere Literatur s. Oppenheimers Handbuch der Biochemie, Bd. I, S. 570 (1908). 
Anders in der Pflanzenphysiologie, wo auch das Vorkommen von Sauerstoffüberträgern viel häufiger, oder wenigstens viel leichter nachweisbar ist. Von älteren Autoren, die sich mit Oxydationsvorgängen in Pflanzen - von der Kohlensäureproduktion abgesehen - befaßt haben, wären außer Schönbein Blanche, ${ }^{1}$ ) van den Broek, ${ }^{2}$ ) Struve, ${ }^{3}$ ) Pfeffer ${ }^{4}$ ) und andere zu nennen. 1883 nennt Hikorokuro Yoshida 5 ) die Substanz, die die Umwandlung von «Urushisäure » in «Oxyurushisäure» veranlaßt, «Diastatic matter».

Besonders die französische Schule hat sich weiter mit den Oxydasen befaßt. Es seien genannt Lindet, ${ }^{6}$ ) vor allem aber Bertrand, 7) Bourquelot. 7) Seit 1902 hat besonders Bach zum Teil gemeinsam mit Chodat die Kenntnis der Oxydasen gefördert.

\section{Die Wirkungsweise der Oxydasen.}

Das Studium der Oxydasen ist zum Teil weniger experimentell als theoretisch betrieben worden. Darum waren auch die Anschauungen über die Oxydasen ziemlichen Schwankungen ausgesetzt. Traube ${ }^{8}$ ) unterschied Oxydationsfermente, «die freien Sauerstoff aufnehmen und ihn auf andere passive Körper übertragen» und Fermente, die «bereits gebundenen Sauerstoff übertragen, d. h. auf der einen Seite Reduktion, auf der andern Oxydation und auf diese Weise einen Platzwechsel, eine Wanderung des Sauerstoffs bewirken». Bourquelot ${ }^{9}$ ) teilte die Sauerstofferreger in Ozon, welches in Organextrakten gelöst oder adsorbiert wird; in Ozonide, die - wie Chinon - oxydierend wirken, aber durch Kochen nicht zerstört werden; in

1) Bull. de Pharm., Bd. VI, S. 579 (1810).

2) J. B. v. Liebig und Kopp, 1849-50, S. 455.

3) Liebigs Ann., Bd. CLXIII, S. 160 (1873).

4) Ber. d. bot. Ges., Bd. III, S. 82 (1889).

5) Journ. of the chem. Soc., Bd. XLIII, S. 442 (1883), Transactions.

6) Le Cidre, S. 150 (1893).

7) Literatur in Green-Wind is ch, Enzyme (1901); Oppenheimer, «Die Fermente> (1903), und Czapek, «Biochemie der Pflanzen» (1905).

$\left.{ }^{8}\right)$ Ges. Abh., S. 385.

9) Journ. Pharm. Chim. (6), Bd. V, S. 465 (1897). 
echte und in indirekte Oxydasen, die nur bei Gegenwart von Wasserstoffsuperoxyd wirken. Grü s s ${ }^{1}$ )bezeichnet als $\alpha$-Oxydasen solche, die bereits ohne Wasserstoffsuperoxydzusatz Guajaklösung bläuen; als $\beta$-Oxydasen solche, die der Gegenwart von Wasserstoffsuperoxyd bedürfen, und als $\gamma$-Oxydasen gleichzeitig hydrolytisch und oxydierend wirkende Stoffe.

Während keine dieser Einteilungen sich weiter hin bewährte, scheint das mit der von Bach und Ghodat eingeführten ziemlich allgemein der Fall zu sein. Sie übernehmen von Linossier ${ }^{2}$ ) den Namen Peroxydasen ${ }^{3}$ ) für Oxydasen, die nur bei Gegenwart von Wasserstoffsuperoxyd wirken, also dasselbe "aktivieren », und nennen Bourquelots "echte Oxydasen», die den molekularen Sauerstoff unter Peroxydbildung aufnehmen und so als Sauerstoffüberträger wirken, $0 x y$ genas en. ${ }^{4}$ )

Schon früher haben Bach, ${ }^{5}$ ) Kastle und Loevenhart, ${ }^{6}$ ) oswie Engler und Wöhler ${ }^{7}$ ) auf die Bildung eines Peroxyds bei den Oxydasen hingewiesen. Kastle und Loevenhart ${ }^{6}$ ) haben auch bereits ausgesprochen, daß sich Wasserstoffsuperoxyd an die Peroxydasen unter Peroxydbildung anlagert, ähnlich wie $\mathrm{H}_{2} \mathrm{O}_{2}$ an $\mathrm{BaO}$ unter Bildung von Bariumperoxydhydrat sich anlagert (vgl. S. 334) und wie dies J. Brode ${ }^{8}$ ) für Eisensalze annimmt. Ganz ebenso wie die Peroxydase Wasserstoffsuperoxyd «aktiviert », vermag sie auch als Peroxyd eine Oxygenase zu aktivieren und ihre Oxydationswirkung zu beschleunigen. ${ }^{9}$ ) Cho dat und Bach zeigten dies, indem sie einen sehr verdünnten Laktariusauszug, der

1) Ber. bot. Ges., Bd. XVI, S. 129 (1898).

2) G. R. Soc. Biol., Bd. V, S. 37 (1898).

3) B. B., Bd. XXXV, S. 1278 (1902).

4) B. B., Bd. XXXVI, S. 606 (1903).

5) G. R., Bd. CXXIV, S. 954 (1897).

6) Am. Chem. Journ., Bd. XXVI, S. 539 (1901).

7) Zeitschr. f. anorg. Chem., Bd. XXIX, S. 1 (1902).

$\left.{ }^{8}\right)$ Zeitschr. f. physik. Chem., Bd. XXXVII, S. 257 (1901). - Vgl. auch Manchot und Wilhelms, B. B., Bd. XXXIV, S. 2479 (1901). - cÜber die Konstitution von solchen Anlagerungsprodukten, E. und W., S. 104-5. Es sei daran erinnert, daß sich die Annahme, es handelt sich um intermediäre Additionsvorgänge, schon bei Kekulé [Liebigs Ann., Bd. CVI. S. 140 (1858)] findet.

9) Chodat und Bach, B. B., Bd. XXXV, S. 3943 (1902). 
allein Gujaktinktur langsam bläut (Oxygenase), mit etwas Kürbisextrakt(Peroxydase) versetzten; die Bläuung trat schnell ein. Dasselbe wurde im hiesigen Laboratorium z. B. mit Auszügen von Champignons (Oxygenase) und Sellerie (Peroxydase) gefunden. In der Regel kommen in den Organismen Peroxydase und Oxygenase vereint vor, sie lassen sich aber $z$. B. durch fraktionierte Alkoholfällung bis zu einemgewissen Grade von einander trennen. ${ }^{1}$ ) Die Annahme, daß sich $\mathrm{H}_{2} \mathrm{O}_{2}$ an die Peroxydasen anlagert, versuchen die Verfasser durch den Nachweis stöchiometrischer Verhältnisse zwischen Peroxydase und $\mathrm{H}_{2} \mathrm{O}_{2},{ }^{2}$ ) sowie dadurch zu stützen, daß die zur völligen Lähmung der Peroxydase erforderlichen Mengen Hydroxylaminchlorhydrat, Hydrazinsulfat, Kaliumcyanid für 1 Molekül $\mathrm{H}_{2} \mathrm{O}_{2}$ resp. $1 / 4$ und 2 Moleküle betragen. ${ }^{3}$ ) $\mathrm{Zu}$ diesen Versuchen ist allerdings zu bemerken, daß bei der Nachprüfung, die R. O. Herzog mit Polotzky anstellte, zumindest die relativen Verhältnisse ganz anders werden, wenn man die gleiche Peroxydase (gereinigtes Produkt aus weißen Rüben) verschiedenen Substraten gegenüber anwendet; jedenfalls handelt es sich bei diesen Experimenten überhaupt um kompliziertere Verhältnisse.

Überblickt man die äußerst zahlreichen Oxydasereagenzien, so findet man angewandt:

1. Jodid in saurer Lösung. Schon Schönbein ${ }^{4}$ ) fand, daß sich angesäuerter, jodkaliumhaltiger Stärkekleister auf Zusatz oxydasehaltiger Extrakte bei Gegenwart von $\mathrm{H}_{2} \mathrm{O}_{2}$ augenblicklich bläut, während dieselbe Reaktion sonst langsam vor sich geht; daß sich in den Extrakten gleichzeitig Stoffe finden, die durch ihre reduzierende Wirkung die Reaktion hemmen können; daß durch Erhitzen oder Vergiftung der Extrakte (oder Blutkörperchen) ihre Wirkung zerstört wird. Später haben

1) Chodat und Bach, B. B., Bd. XXXVI, S. 606 (1903); vgl. auch Die Zerlegung der Tyrosinase, Bach, B. B., Bd. XLI, S. 216 (1908).

2) B. B., Bd.XXXVII, S.1346, 3785 (1904); Bd.XXXVIII, S.1878 (1905).

3) B ach, B. B., Bd. XL, S. 3185 (1907).

4) Siehe Abh. d. Basler Naturf.-Ges., Bd. I-V inkl. - Z. f. Biol., Bd. I-IV inkl. - Kgl. Bayer. Akad. d. Wissensch., 2. Kl., Bd. VIII, 1. Abt. 
sich Wurster, ${ }^{1}$ ) ferner R. Chodat und A. Bach ${ }^{2}$ ) u. a. mit derselben Reaktion beschäftigt.

2. Phenole. Man findet die meisten Reagenzien dieser Gruppe in der Arbeit von 0. und R. Adler ${ }^{3}$ ) angeführt; obwohl sich die Reaktionen dort auf den Blutfarbstoff beziehen, dürfen sie doch hier angeführt werden, da Oxydasen dasselbe Verhalten zeigen. Es handelt sich um Brenzkatechin, Orcin, Hydrochinon, Pyrogallol, Phloroglucin, Naphtolen, Gujakol, ${ }^{4}$ ) Guajak, Guajacin, Protokatechusäure, Gallussäure, ${ }^{5}$ ) Guajakonsäure, Aloin ${ }^{6}$ ) u. a.

3. Amine. Die meisten Beispiele finden sich wieder bei 0 . und R. Adler angeführt. Anilin und seine Substitutionsprodukte, p-Toluidin, o-, m-, p-Phenylendiamin und Substitutionsprodukte, Benzidin, Tolidin, Amidophenole, Ursol-D, ${ }^{7}$ ) Pyramidon ${ }^{8}$ ) u. a.

4. Leukobasen resp. Gemische ihrer Bildner. ${ }^{9}$ ) Die Leukobasen von Indigotin und Methylenblau, der Malachitgrünreihe (Malachitgrün, Brillantgrün, Säuregrün), ${ }^{10}$ ) die Rosanilinderivate (Dahlia, Methylviolett, Krystallviolett), Phenolphthalein ${ }^{11}$ ) und sein Äthylester, ${ }^{12}$ ) Leukorosolsäure ${ }^{13}$ ) usw., die Bildung von Indophenol aus $\alpha$-Naphtol und Paraphenylendiamin in sodaalkalischer Lösung, ${ }^{14}$ ) statt Paraphenylendiamin kann auch Dimethylparaphe-

1) B. B., Bd. XXI, S. 1526 (1888).

?) B. B., Bd. XXXV, S. 2466, 3943 (1902); ferner Czyhlarz und Fürth, Hofmeisters Beitr., Bd. X, S. 358 (1907). - Batelli und Stern, Biochem: Zeitschr., Bd. XIII, S. 44 (1908). - Engler und W eiss berg, B. B., Bd. XXXIIl, S. 1104 (1900).

3) Diese Zeitschrift, Bd. XLI, S. 59 (1904).

4) Soc. Biol., Bd. XLVI, S. 896 (1896); Bd. L, S. 381.

5) Cizyhlarz und Fürth, l. c. S. 365 .

6) Schaer, Z. f. anal. Chem., Bd. XLII, S. 7 (1903).

7) Utz, Chem. Ztg., Bd. XXVI, S. 1121 (1902). - Chlopin, Chem. Zentralbl., 1902, Bd. II, S. 157. - Arnold u. Mentzel, B. B., Bd. XXXV, S. 2902 (1902).

$\left.{ }^{8}\right)$ Kobert, Chem. Zentralbl., 1903, Bd. II, S. 262. - Rudillon, das., 1903, Bd. I, S. 642 .

9) Siehe S. 346 (Indophenol).

10) Diese Zeitschrift, Bd. XLI, S. 63 (1904).

1i) Castle und Shedd, Am. chem. Journ. Bd. XXVI, S. 527 (1901).

12) J. H. Kastle, Chem. Zentralbl., 1906, Bd. I, 1555.

13) Das.

14) Röhmann und Spitzer, B. B., Bd. XXVIII, S. 567 (1894). 
nylendiamin angewandt werden; «ebenso gelingt mit Leichtigkeit die Bildung der Indamine, z. B. des Toluylenblaues aus Metatoluylendiamin und Paraphenylendiamin, bezw. Dimethylparaphenylendiamin, Bindschedlers Grün aus Dimethylparaphenylendiamin und Dimethylanilin. Auch die Eurhodine lassen sich in derselben Weise erhalten*. ${ }^{1}$ ) Natürlich sind hier eine Unzahl von Variationen möglich, die zu mehr oder weniger gutem Resultat führen, z. B. ließ sich in unserem Laboratorium Fuchsin aus einem Gemisch von o- und p-Toluidin und Anilin, Wasserstoffperoxyd und Rübenperoxydase herstellen usw.

5. Die fünfte Gruppe der Oxydasereagenzien bilden gewisse Benzolderivate, die bei der Oxydation unter Wasseraustritt in Diphenylderivate umgewandelt werden, wie Vanillin, das in Dehydrodivanillin, ${ }^{2}$ ) Eugenol, das in Dehydrodieugenol, ${ }^{3}$ ) Isoeugenol, das in Dehydrodiisoeugenol, ${ }^{4}$ ) Thymol, das in Dehydrodithymol ${ }^{5}$ ) verändert wird.

Von den genannten Reagenzien hat man auch eine Reihe zu quantitativen Messungen zu verwenden gesucht. So bemühte sich Laborde,$^{6}$ ) die Färbung der Guajaktinktur kolorimetrisch zu messen, eine Methode, die Alliot und PozziEscot 7) als unbrauchbar erkannten. Auch die kolorimetrische Methode von Slow z off, ${ }^{8}$ ) der die Indophenolreaktion anwandte, und die von Kastle und Shedd, ${ }^{9}$ ) welche zu Phenolphthalein griffen, ist nicht empfehlenswert, da selbsttätig an der Luft indirekte Autoxydation eintritt. Dasselbe fanden R. O. Herzog und Polotzky, als sie nach dem Vorschlag von Czyhlarz und $\mathrm{Fürth}{ }^{10}$ ) Leukomalachitgrün anwandten; dagegen bewährte

1) Das., S. 570.

2) Bourquelot-Marchartier, C. R., Bd. CXXXVIII, S. 1432 (1904); Journ. pharm. chim. (6), Bd. XX, S. 5; ferner Lerat, C. R. de Soc. Biol., Bd. LV, S. 1325 (1902); Journ. pharm. chim. (6), Bd. XIX, S. 10 (1904).

s) C. R., Bd. CXLV, S. 1413 (1908).

4) Das., Bd. CXLVII, S. 247 (1908).

5) Journ. pharm. chim. (6), Bd. XXVI, S. 487 (1907).

ๆ C. R., Bd. CXXVI, S. 536 (1898).

7) Chem. Zentralbl., 1902, Bd. II, S. 305.

8) Diese Zeitschrift, Bd. XXXI, S. 227 (1900).

9) Am. chem. Journ., Bd. XXVI, S. 26 (1901).

10) Hofmeisters Beitr., Bd. X, S. 370 (1907). 
sich ihnen besser die Oxydation von Leukobrillantgrün zum Farbstoff; vermutlich ist die Herkunft des Farbstoffs für seine Oxydierbarkeit nicht gleichgültig. Bach verwandte die Jodidoxydation, ${ }^{1}$ ) Bach und Chodat die Umwandlung von Pyrogallol in Purpurogallin. ${ }^{2}$ ) Besser als die letzte Methode erschien R. 0. Herzog und A. Meier noch die Oxydation des Vanillins in Dehydrodivanillin, das etwa zu 80\% umgewandelt wird, als gravimetrische Methoden brauchbar. Batelli und Stern $^{3}$ ) verwenden Ameisensäure, die allerdings auch ohne Peroxydase merklich verbrannt wird.

Außer den genannten von den Oxydasen allgemein angreifbaren Stoffen (wobei allerdings zwischen den Oxydasen verschiedener Herkunft bereits wesentliche Unterschiede auftreten, wie z. B. auch Versuche von R. O. Herzog mit Ripke gezeigt haben), sind aber auch auf spezielle Oxydasen abgestimmte Stoffe bekannt geworden. So sei an die Aldehydase Jakobys , ${ }^{4}$ ) an die Adrenalinoxydase von Neuberg, ${ }^{5}$ ) vor allem an die Tyrosinase ${ }^{6}$ ) erinnert, von der sowohl Abderhalden und Guggenheim, 7) wie Ghodat $^{8}$ ) gezeigt haben, daß sie nur eine bestimmte stereochemische Konfiguration des Substrates angreifen.

Der Chemismus gewisser, bisher noch nicht genauer bekannter, als Oxydasewirkungen beschriebener Reaktionen, wie der Laccase,$^{9}$ ) der Oenoxydase, ${ }^{10}$ ) der Gummasen, ${ }^{11}$ ) der im Lichte

1) B. B., Bd. XXXVII, S. 3785 (1904).

$\left.{ }^{2}\right)$ B. B., Bd. XXXVII, S. 1342, 2434 (1905),

$\left.{ }^{3}\right)$ Biochem. Z., Bd. XIII, S. 44 (1908).

$\left.{ }^{4}\right)$ Diese Zeitschrift, Bd. XXX, S. 135 (1900).

5) Biochem. Z., Bd. VIII, S. 383 (1908).

${ }^{6}$ B Bourquelot und Bertrand, Journ. pharm. chim. (6), Bd. III, S. 177 (1896); weitere Literatur s. Czzapek, Biochem. d. Pflanz., Bd. II, S. 478, 462 (1905); Über die Oxydation von Phenylalanin, das., S. 183, Anm. 3 ; S. 462 , Anm. 5.

$\left.{ }^{7}\right)$ Diese Zeitschrift, Bd. LIV, S. 331 (1907).

$\left.{ }^{8}\right)$ Arch. sc. phys. et nat., Bd. XXVI, S. 112 (1907).

9) Yoshida, Journ. Chem. Soc., Bd. XLIII, S. 472 (1883). Bertrand, C. R., Bd. GXVIII, S. 215 (1894) usw.

10) B a uffard, C. R., Bd. CXVIII, S. 827 (1894); Bd. CXXIV, S. 706 (1897). - Gouirand, das., Bd. CXX, S. 887 (1895) usw.

11) Tchirch u.Stevens, Arch. f. Pharm., Bd. CCXLIII, S. 504(1905). 
wirksamen Luciferase ${ }^{1}$ ) und Purpurase ${ }^{2}$ ) u. a., ist natürlich vorläufig nicht festzustellen.

Vermutlich hat es zu gewissen Zweifeln geführt, daß bis vor einiger Zeit gerade diese letztgenannten Vorgänge vorwiegend untersucht wurden. Dazu kam noch, daß nach Bertrand ${ }^{3}$ ) Mangansalze, nach Sarthou $\left.{ }^{4}\right)$ mitunter auch Eisensalze eine erhebliche Rolle spielen, daß die Oxydasen gegen Siedehitze wenig empfindlich sind, 5 ) was gegen ihre kolloidale Natur zu sprechen scheint, ${ }^{6}$ ) daß die Wirksamkeit im besonderen bei Anwendung von $\mathrm{H}_{2} \mathrm{O}_{2}$ statt 0 xygenase abnimmt, und endlich, daß diesen Oxydasen im Gegensatz zu den bekannten, echten Fermenten scheinbar keine wesentliche biologische Bedeutung zukam. Höchstens gewisse Farbstoffbildungen konnten auf ihre Wirkung zurückgeführt werden. ${ }^{7}$ ) Die Wichtigkeit der Oxydasen ist mit dem Nachweis gestiegen, daß eine ganze Reihe von Aminosäuren und Peptiden angreifbar sind. ${ }^{8}$ ) Was das Mangan betrifft, zeigte Bertrand selbst ${ }^{9}$ ) sowie andere, ${ }^{10}$ ) daß $\mathrm{Mn}$ bloß

1) Dubois, Soc. Biol., Bd. LIV, S. 82 (1902).

9) Dubois, das., Bd. LV, S. 230 (1903). - Es muß hier noch daran erinnert werden, daß gerade Oxydations-Reduktionsvorgänge sehr häufig photochemisch beeinflußbar sind. - Hier sei noch an die Arbeiten von Tappeiner und Jodlbauer (Leipzig 1907, siehe bes. D. Arch. f. klin. Med., Bd. LXXXII (1905), u. Biochem. Zeitschr., Bd. VIII, 1908) erinnert. Siehe auch W. Ostwald, das., Bd. VI, S. 409 (1907) u. a.

s) C. R., Bd. GXXIV, S. 1032, 1355 (1897); s. auch Denigés, das., Bd. CXXX, S. 32 (1900).

4) Journ. pharm. chim. (6), Bd. XI, S. 482 ; Bd. XII, S. 104;

Bd. XIII. S. 464 .

5) Woods, zit. nach Oppenheimers Fermente, S. 366 (1903); Bach u. Tscherniak, B. B., Bd. XLI, S. 2349 (1908); B. B., Bd. XXXVI, S. 603 (1903), u. a. auch durch eigene Versuche bestätigt.

6) Bach u. Tscherniak, «Über Dialyse», l. c. S. 2347.

7) Vgl. dazu Fürth und Schneider, Hofmeisters Beitr., Bd. I, S. 229 (1901). - Gessard, Ann. Pasteur, Bd. XV, S. 593 (1901) usw.

8) Hier sei auch die Angabe von N. Si eber (Chem. Zentralbl., 1903, Bd. I, S. 408) angeführt, daß Oxydasen Toxine zu zerstören vermögen.

9) 1. c.

10) Bach und Tscherniak, 1. c.; vgl. ferner den Versuch der Trennung von Salz und Oxydase durch Kapillaranalyse von Raudnitz, Monatsschr. f. Kinderh., Bd. I, Nr. 5 (1903). 
wie ein sogenanntes (1o-Enzym ${ }^{1}$ ) als Aktivator ${ }^{2}$ ) wirke. Die weniger kolloidale Natur kann mit der möglichen Natur der Stoffe als Salze ${ }^{3}$ ) zusammenhängen. Die Abnahme der Wirksamkeit wurde bereits früher erklärt. Wir dürfen danach als das wahrscheinlichste aussprechen, daß wenigstens die Mehrzahl der bisher erwähnten sogenannten Oxydationsfermente nicht Katalysatoren, sondern Induktoren resp. Gemische von Induktoren und Aktoren vorstellen, also ähnlich, wie etwa ein Gemisch von $\mathrm{H}_{2} \mathrm{O}_{2}$ mit Ferrosulfat wirkt, von dem bereits Schönbein bemerkt hat, daß es dieselben Reaktionen wie die Oxydasen zu veranlassen vermag. ${ }^{4}$ ) Dasselbe Gemisch verwandelt spielend Benzol zu Phenol und ruft noch andere Reaktionen hervor, die $z u$ den längstbekannten der Oxydationswirkungen des Tierkörpers gehören, wie R. O. Herzog fand.

Schon im theoretischen Teil ist gezeigt worden, daß es eigentlich mehr eine Frage zufälliger Bedingungen ist, ob man eine Reaktion als katalytische oder induzierte beobachtet, während sich das Wesen beider Reaktionsbeeinflussungen sehr häufig nicht unterscheidet. Nur eine genaue und vollständige physikalisch-chemische Analyse, die sich auf sämtliche an der Reaktion beteiligten Bestandteile erstreckte, wäre imstande, vollen Aufschluß über den Tatbestand zu geben. Liegt eine solche schon für die relativ einfachen, absolut aber bereits sehr komplizierten, bisher angeführten Fälle noch nicht vor, ${ }^{5}$ ) so ist sie noch viel weniger $\mathrm{zu}$ erwarten für andere Vorgänge, die man vorläufig auch häufig als durch Oxydationsfermente verursacht anspricht. Hierher gehören die Purinoxydasen, ${ }^{6}$ ) das essigsäure-

1) Siehe M. Jakoby, Handb. d. Biochem., Bd. II, 1. H., S. 152.

2) Bredig, Biochem. Z., Bd. VI, S. 317 (1907). - Vgl. auch die «Acceleratoren von J. H. Kastle, Am. Chem. Journ., Bd. XL, S. 251 ; Ghem. Zentralbl., 1908, Bd. II, S. 2026.

3) Euler und Bolin, Diese Zeitschrift, Bd. LVII, S. 80 (1908).

4) Vgl. Nencki und Sieber, J. f. prakt. Chem.. Bd. XXVI, S. 1 (1882). - Leeds, B. B., Bd. XIV, S. 975. - Diese Vorgänge werden weiter verfolgt.

5) Bei dem von Burian, Diese Zeitschrift, Bd. XLIII, S. 497 (1905), studierten Fall liegen die Verbältnisse an sich zu kompliziert.

6) Xanthoxydase, Guanase, Adenase; Spitzer, Pflügers Arch., 
bildende Ferment, ${ }^{1}$ ) die Acidoxydase, ${ }^{2}$ ) Vorgänge, an die sich - wenn auch über das oxydierende Substrat so gut wie nichts bekannt ist - ungezwungen anschließen: die Spaltung von Razematen im Tierkörper, ${ }^{3}$ ) ferner die Oxydation von Propylalkohol, Glykol, Glycerin, Arabinose, Lävulose, Dextrose, Galaktose, Maltose, Saccharose, Mannit, Erythrit, Raffinose, ${ }^{4}$ ) weiterhin ist hier zu erinnern an die Wirkung des Sorbosebakteriums ${ }^{5}$ ) (Sorbit zu Sorbose, Mannit zu Lävulose), des Mikrococcus chinicus $^{6}$ ) (Chinasäure zu Protokatechusäure), an die Umwandlung von Isoleucin in Amylalkohol ${ }^{7}$ ) usw.

Wie gesagt, weiß mąn bei diesen Vorgängen so gut wie

Bd. LXXVI, S. 192 (1899). - Wiener, Arch. f. exp. Path., Bd. XLII, S. 375 (1899); weitere Literatur bei Abderhalden, Lehrb. d. physiol. Chem., 2. Aufl. (1909), S. 393-96; vgl. auch Lehmann, Diese Zeitschrift, Bd. IX, S. 563 (1885); K. Shig a, das., Bd. XLII, S. 502 (1904). Uricolytisches Ferment Wiechowski und Wiener, Hofmeisters Beitr., Bd. IX, S. 247 (1907). - Schittenhelm, Diese Zeitschrift, Bd. XLV, S. 161 (1905) usw.

1) Buchner und Meis enheimeir, B. B., Bd. XXXVI, S. 634 (1903), und Liebigs Ann., Bd. CCCXLIX, S. 140 (1906).

8) R. 0. Herzog und Meier, Diese Zeitschrift, Bd. LVII, S. 35 (1908); Bd. LIX, S. 38 (1909).

s) Oxydation von Weinsäuren, Brion, Diese Zeitschrift, Bd. XXV, S. 283 (1898). - Kohlehydrate, Neuberg und Wohlgemuth, B. B., Bd. XXXIV, S. 1745 (1901); Diese Zeitschrift, Bd. XXXV, S. 41 (1902); Bd. XXXVII, S. 530 (1903). - Aminosäuren, Wohlgemuth, B. B., Bd. XXXVIII, S. 2064 (1905). - Abderhalden und Samuely, Diese Zeitschrift, Bd. XLVII, S. 346 (1906). - Embden, Hofmeisters Beitr., Bd. XI, S. 348 (1908).

4) Brown, Journ. Chem. Soc., Bd. XLIX, S. 112 (1886). - Seifert, Zentralbl. f. Bakt. (II), Bd. III, S. 337 (1893); bes. Henneberg, das., S. 223; Bd. IV, S. 14; Bd. LXVII, S. 138, 933 (1898); zit. nach O. Emmerling, Biochem. Zentralbl., Bd. II, S. 385 (1904). - Nach Seifert, l. c., oxydieren Essigbakterien Alkohole mit verzweigten Ketten weniger gut als mit normalen.

5) Bertrand, C. R., Bd. CXXII, S. 9090; Bd. CXXVI, S. 762, 842, 984; Bd. CXXVII, S. 124, 729; Vincenz u. Delachanal, das., Bd. CXXV, S. 716; Kling, das., Bd. CXXVIII, S. 244; Bd. CXXIX, S. 1252 usw.

6) Loew, B. B., Bd. XIV, S. 450 (1881). - Emmerling und Abderhalden, Zentralbl. f. Bakt. (II), Bd. X, S. 337 (1903).

7) F. Ehrlich, Biochem. Z., Bd. II, S. 71 (1906); das., Bd. I, S. 8 (1906). 
nichts über das die Oxydation vermittelnde Prinzip. Man erkennt nur, daß es sich um in ihrer Wirkung mehr oder weniger speziell abgestimmte Agenzien handeln muß und daß diese Agenzien zu den aus der Chemie gemeinhin bekannten 0xydationsmitteln nicht gehören können. Soll es sich nicht um Verbindungen handeln, die gänzlich verschieden von dem sind, was wir bisher aus der Chemie gelernt haben, so gibt es bloß eine Deutung, daß es sich nämlich um Superoxyde handelt, die den Sauerstoff abgeben, Superoxyde, die sich primär durch Autoxydation gebildet haben. Dabei sind für den Vorgang der Sauerstoffübertragung vor allem die Verbindungen ${ }^{1}$ ) wesentlich, welche die oxydablen Stoffe im Organismus eingehen; Verbindungen, die sie einmal in den chemischen Umsatz des Lebens hineinreißen können, indem sie sie ungesättigter, dissoziationsfähiger machen, und Verbindungen, die sie ein andermal der Umwandlung zu entziehen vermögen durch Absättigung freier Valenzen, durch gewaltsame Verankerung (vgl. S. 344).

$\mathrm{Zu}$ der ersten Gruppe von solchen Vorgängen gehört offenbar die so häufige $0 x y d a t i o n$ von Methylgruppen. So zeigt sich z. B., daß die Methylgruppen des Coffeins im Organismus des Hundes anders als in dem des Kaninchens angegriffen werden. Beim Hunde ist die 3-Methylgruppe am meisten, beim Kaninchen am wenigsten angreifbar, soda $B$ im ersten Fall hauptsächlich 1,3-Dimethyl-2,6-Dioxypurin (Theophyllin), und 3-Methyl-2,6-Dioxypurin, im zweiten 1,7-Dimethyl2,6-Dioxypurin (Paraxanthin), 1-Methyl- und 7-Methyl-2,6-Dioxypurin im Harn sich finden. ${ }^{2}$ ) Solche Vorgänge lassen sich

1) Anderseits erklärt sich wohl das Nichtzustandekommen solcher Verbindungen, warum sonst leicht angreifbare Stoffe den Tierkörper wiederum ohne weiteres passieren, wie z. B. die höheren Alkohole: Erythrit, Quercit, Mannit, v. Mering, Pflügers Arch., Bd. XIV, S. 274 (1877); Pohl, Arch. f. exp. Path., Bd. XXXVII, S. 413; Luchsinger, Diss. Zürich 1875, S. 48, u. a. Stoffe, so gewisse Antipoden der Aminosäuren, Magnus-Levy, Münch. med. Wochenschr., 1905, S. 2168 usw.

2) Albanese, Arch. f. exp. Path., Bd. XXXV, S. 449 (1895), und B. B., Bd. XXXII, S. 2280 (1899). - Bondzyński und Gottlieb, Archiv f. exp. Path., Bd. XXXVI, S. 45 (1895); Bd. XXXVII, S. 385 (1896). Krüger, B. B., Bd. XXXII, S. 2818 (1899). 
doch wohl nur durch das Vorkommen in beiden Tierarten verschiedener, an der Reaktion aktiv beteiligter Agenzien erklären. Dabei darf nicht vergessen werden, daß man allen Grund hat, bei der Oxydation überhaupt Anlagerung des Oxydationsmittels an den Acceptor anzunehmen. ${ }^{1}$ ) Vermutlich verlaufen diese Oxydationen in Stufen, wenigstens ist in einigen Fällen die Oxydation von $-\mathrm{CH}_{3} \mathrm{zu}-\mathrm{CH}_{2} \mathrm{OH}$ gefunden worden; $-\mathrm{CH}_{2} \mathrm{OH}$ wird dann natürlich leichter in $\mathrm{CO}_{2} \mathrm{H}$ oxydiert.

Toluol wird zur Benzoesäure oxydiert, ${ }^{2}$ ) Äthylbenzol, Propylbenzol verhalten sich ebenso, ${ }^{3}$ ) Xylol erscheint als Toluylsäure, $\left.{ }^{4}\right)$ Pseudocumol als p-Xylylsäure, p-Cymol als Cuminsäure, ${ }^{5}$ ) Mesitylen als Mesitylensäure ${ }^{6}$ ) im Harn. m-Kresol scheint den Körper unverändert zu verlassen, während die p-Verbindung in p-Oxybenzoesäure übergeht und o-Kresol zu Hydrotoluchinon umgewandelt wird. ${ }^{7}$ ) Dimethyltoluidin wird zur p-Dimethylaminobenzoesäure, ${ }^{8}$ ) ebenso die Acetylverbindungen der Toluidine, so wird p-Acetoluidid zu p-Acetylaminosäure. ${ }^{9}$ ) p-Nitrotoluol wird zur p-Nitrobenzoesäure oxydiert, analog verläuft die Reaktion mit der $0-V e r b i n d u n g$, daneben erscheint aber o-Nitrobenzylalkohol als ein Zwischenprodukt. ${ }^{10}$ ) Die 3 Chlortoluole gehen in die entsprechende Chlorbenzoe-

1) Manchot, Verhandl. d. phys.-med. Ges. Würzburg, Bd. XXXIX, S. 215 (1908).

2) Schultzen und Naunyn, Du Bois-Reymonds Archiv (1867), S. 349, 353. - Benzylamin wird nach Schmiedeberg, Arch. f. exp. Path., Bd. XIV, S. 288 (1881), zunächst desamidiert, vgl. S. 348, Anm. 8, und dann zur Benzoesäure oxydiert.

3) Neubauer, Arch. f. exp. Path., Bd. XLVI, S. 133 (1901).

4) Schultzen und Naunyn, l. c.

5) Jacobson, B. B., Bd. XII, S. 1512 (1879); vgl. auch B. B., Bd. V, S. 749 (1872); Arch. f. exp. Path., Bd. I, S. 65 (1873); Diese Zeitschrift, Bd. XXXVI, S. 452 (1902), u. das., Bd. IV, S. 336 (1880).

6) Nencki, Arch. f. exp. Path., Bd. I, S. 420 (1873).

7) Ba umann und Herter, Diese Zeitschrift, Bd. I, S. 264 (1877/78).

- Preusse, das., Bd. III, S. 329 (1878); Bd. V, S. 57 (1880),

8) Hofmeister Beitr., Bd. VII, S. 433 (1906).

$\left.{ }^{9}\right)$ Jaffé und Hilbert, Diese Zeitschrift, Bd. XII, S. 295 (1888).

10) Jaffé, B. B., Bd. VII, S. 1673 (1874); Diese Zeitschrift, Bd. II, S. 47 (1878). 
säure über, ähnlich bei den Bromtoluolen. ${ }^{1)}$ Acetophenon geht in Benzoesäure über, ${ }^{2}$ ) p-Methylchinolin wird zum Teil in p-Chinolincarbonsäure umgewandelt, während die anderen Chinolinderivate meist nicht im Harn erscheinen. ${ }^{3}$ ) Pikolin wird zur Pyridincarbonsäure oxydiert, ${ }^{4}$ ) $\alpha$-Methylpiperidin zu $\alpha$-Piperidincarbonsäure. ${ }^{5}$ ) Theophyllin erscheint als 3-Methylxanthin. ${ }^{6}$ )

Von denselben Gesichtspunkten ist auch die $0 \mathrm{xydation}$ von Kohlenwasserstoffen zu Phenolen zu betrachten. Nach Einnahme von Benzol wird bekanntlich Phenol aus dem Harn gewonnen. ${ }^{7}$ ) Naphthalin wird zu Naphthol, ${ }^{8}$ ) Diphenylmethan, zu p-Oxydiphenylmethan, Diphenyl zu p-Oxydiphenyl, ${ }^{9}$ ) Chinolin zu 5, 6 Chinolinchinon, ${ }^{10}$ ) Indol zu Indoxyl, Skatol zu Skatoxyl. ${ }^{1}$ )

1) Hildebrandt, Hofmeisters Beitr., Bd. III, S. 365 (1903). Preusse, Diese Zeitschrift, Bd. V, S. 57 (1880).

2) Journ. f. prakt. Chem., N. F., Bd. XVIII, S. 288 (1878).

s) Gohn, Diese Zeitschrift, Bd. XX, S. 210 (1894).

4) R. Cohn, das., Bd. XVIII, S. 112 (1893).

s) Hildebrandt, Arch. f. exp. Path., Bd. XLIV, S. 278 (1900).

o) Krüger und Schmidt, Diese Zeitschrift, Bd. XXXVI, S. 1 (1902). - Es darf nicht unerwähnt bleiben, daß, wie ja bekannt, in anderen Fällen die Methylgruppe einfach abgespalten wird, wie bei Theobromin, das in beide möglichen Monomethyldioxypurine verändert wird [Albanese, Arch. f. exp. Path., Bd. XXXV, S. 449 (1895); Bondzyński und Gottlieb, das., Bd. XXXVI, S. 45 (1895); Bd. XXXVII, S. 385 (1896); Krüger und Schmidt, B. B., Bd. XXXII, S. 2677 (1899); Diese Zeitschrift, Bd. XXXII, S. 104 (1900)]. Wieder in anderen Fällen ist der Organismus sogar imstande, Methylierungen vorzunehmen; so wird Pyridin in Methylpyridylammoniumbase [His, Arch. f. exp. Path., Bd. XXII, S. 253 (1887); R. Gohn, Diese Zeitschrift, Bd. XVIII, S. 112 (1893)], p-Thymodinpiperidid, desgl. die o-Verbindung, p-Kresol, Thymolcarvakrol methyliert [Hildebrandt, Arch. f. exp. Path., Bd. XLIV, S. 278 (1900); Diese Zeitschrift, Bd. XLIII, S. 249 (1904)].

7) Schultzen und Naunyn, l. c. - Baumann und Herter, Diese Zeitschrift, Bd. I, S. 264 (1877/78). - Nencki und Giacosa, das., Bd. IV, S. 339 (1880).

8) Lešnik, Arch. f. exp. Path., Bd. XXIV, S. 167 (1887).

9) Klingenberg, Diss. Rostock 1891.

10) Arch. f. exp. Path., Bd. LV., S. 27 (1906).

11) Baumann und Brieger, Diese Zeitschrift, Bd. III, S. 254 (1879). 
Von Interesse erscheint auch die 0xydation von Santonin ${ }^{1}$ ) zu Oxysantonin.

Auch die Oxydation von Seitenketten an Kohlenstoffringen könnte man hier anführen. Es ist bei dieser Gelegenheit wie bei einer ganzen Reihe von sogenannten Verbrennungen im Organismus wieder daran zu erinnern, daß zunächst gebildete, leicht oxydable Oxydationsprodukte natürlich solange weiter vom Sauerstoff angegriffen werden, bis ein stabiles Produkt gebildet ist. Sehr reichlich finden sich Beispiele dafür auch in der Literatur über die Oxydation von Säuren. In anderen Fällen darf übrigens nicht vergessen werden, daß die Umwandlung im Organismus auch eine Assimilation bedeuten kann.

Über welche außerordentlichen chemischen Fähigkeiten der Organismus verfügt, zeigen noch einige hier anzuführende Reaktionen; so die Oxydation des Wasserstoffs zu W'asser durch Bakterien, ${ }^{2}$ ) die Oxydation von Ammoniak zu Stickstoff und zu Nitrat, ${ }^{3}$ ) von Schwefelwasserstoff zu Schwefel und Sulfat ${ }^{4}$ ) durch Bakterien. Letzterem Vorgang ähnlich ist die Oxydation von Äthylmerkaptan im tierischen Organismus, ${ }^{5}$ ) von dem man weiß, ${ }^{6}$ ) daß es erst durch starke Salpetersäure zu Äthansulfonsäure umgewandelt wird. Ihr entspricht die der Thioglykolsäure. ${ }^{7}$ ) Noch merkwürdiger erscheint die Verbrennung des Methans zur Kohlensäure ${ }^{8}$ ) durch Mikroorganismen, der die Zerstörung von Vaselin, Kohlenwasserstoffen der Paraffinreihe im tierischen Organismus ${ }^{9}$ ) entspricht. Endlich sei hier noch die Oxydation von Kohlenoxyd zur Kohlensäure ${ }^{10}$ ) angeführt. ${ }^{11}$ )

1) Jaffé, Diese Zeitschrift, Bd. XXII, S. 538 (1896); Chem. Zentralblatt, 1899, Bd. I, S. 995.

2) Kaserer, Zentralbl. f. Bakt. (II), Bd. XVI, S, 681 (1906).

$\left.{ }^{3}\right)$ Winogradsky, Ann. Pasteur (1890/91).

4) Winogradsky, Beitr. z. Morph. u. Physiol. d. Bakt., 1. Heft (1888).

5) Smith, Pflügers Arch., Bd. LVII, S. 418 (1894).

6) Prinz, Liebigs Ann., Bd. CGXXIII, S. 377 (1884).

7) Smith, Diese Zeitschrift, Bd. XVII, S. 459 (1893).

8) Soehngren, Zentralbl. f. Bakt. (II), Bd. XV, S. 513 (1906).

9) Sobieranski, Arch. f. exp. Path., Bd. XXXI, S. 329 (1893).

10) Kaserer, Zentralbl. f. Bakt. (II), Bd. XVI, S. 681 (1906).

1i) Anmerkungsweise seien hier auch einige biologische Reduktionsvorgänge angeführt, die ja mit den Oxydationen enge verknüpft sind. 


\section{Die Katalasen.}

Anhangsweise mögen hier noch die Katalas en besprochen werden.

Wasserstoffsuperoxyd bildet sich bei der Autoxydation, wie schon besprochen wurde, bei zwei Gruppen von Vorgängen:

Von den hierhergehörigen Reaktionen ist vor allem die Reduktion von Farbstoffen häufig untersucht worden; es seien angeführt die bekannte Arbeit von P. Ehrlich, .Das Sauerstoffbedürfnis des Organismus», Berlin 1883 ; die Arbeiten von Herter, Diese Zeitschrift, Bd. XLII, S. 493 (1904). Weitere Literatur bei Abderhalden, Lehrbuch, S. 440. Es möge hier aber nicht unerwähnt sein, daß nach Versuchen, die R. O. Herzog mit A. Polotzky angestellt hat, bereits die Oxydasen bei Gegenwart von äußerst wenig Wasserstoffsuperoxyd durch $0 x y d a t i o n d i e$ Farbstoffe $z u$ entfärben vermögen. Es ist also notwendig, stets $z u$ untersuchen, ob in der Tat eine Reduktion zur Leukobase oder eine weitere Oxydation des Farbstoffs stattgefunden hat. Als weitere Reduktionsreaktionen seien angeführt: Benzaldehyd wird zum Teil zu Benzalkohol reduziert [C: Siebert, Diss. Königsberg 1901; R. Cohn, Diese Zeitschrift, Bd. XIV, S. 203)]. Chloralhydrat wird analog zum Trichloräthylalkohol [v. Mering, Diese Zeitschrift, Bd. VI, S. 480 (1882); Külz, Pflügers Arch., Bd. XXVIII, S. 506 (1882)]. Butylchlorhydrat verhält sich analog, ebenso Bromalhydrat (Maraldi, Chem. Zentralbl., 1903, Bd. I, S. 781). Diese Vorgänge sind wohl so zu erklären, daß die reaktionsfähige Aldehydgruppe gegen die Oxydation geschützt wird und zwar geht diese Schutzreaktion schneller als die Oxydation vor sich. Auch die Ketone können reduziert werden; so geht Chloraceton in Isopropylalkohol über [Sundwik, J. B. f. Tierchem., Bd. XVI, S. 76 (1886)]. Chinasäure wird zu Benzoesäure reduziert [Lautemann, Ann. d. Chem., Bd. CXXV, S. 9 (1863)]. Chinon wird zu Hydrochinon (Wöhler und Frerichs, Liebigs Ann., Bd. LXV, S. 335 (1848); O. Schulz, Diss. Rostock, 1892; Cohn, Diss. Königsberg, 1893). Thujon geht in den Kohlenwasserstoff $\mathrm{C}_{10} \mathrm{H}_{14}$ über [Hildebrandt, Arch. f. exp. Path., Bd. XLV, S. 110 (1900); Fromm und Hildebrandt, Diese Zeitschrift, Bd. XXXIIl, S. 579 (1901)]. Chlorate werden nicht, Bromate wenig, Jodid reichlich reduziert. Jodoanisol wird zu Jodanisol [Röhmann, Biochem. Zentralbl., Bd. III, S. 688, 1905)]. Nitrobenzol zu Anilin [Abelous und Gérard, C. R., Bd. XIII. S. 420 (1900)]. Pikrinsäure zu einem Aminoderivat (Walko). Nitrobenzol zu Aminophenol, ebenso Nitrophenol (Diese Zeitschr., Bd. XLVI, S. 497 [1906]). Weiters sei noch an die Reduktion von Arsensäure, Tellursäure und Selensäure durch Schimmelpilze (Giglioni, Annuar. Sz. sub. d'aggricolt. Portici (1880); Biginelli, Atti d. R. Accad. de Lincei, Rom 1900, V. 9, 
1. durch direkte Autoxydation gebildetes Peroxyd kann sich dann mit Wasser hydrolysieren unter sekundärer Bildung von Peroxydhydrat bezw. Wasserstoffsuperoxyd;

2. molekularer Saurerstoff lagert sich an reaktionsfähigen Wasserstoff und bildet damit Wasserstoffsuperoxyd. ${ }^{1}$ )

Diese Vorgänge müssen im Organismus ebenso stattfinden wie außerhalb. Wenn auch Wasserstoffsuperoxyd in sehr schwachen Konzentrationen nicht giftig ist, muß es im Organismus doch da zerstört werden, wo es sich in größeren Mengen bildet, und das geschieht durch die Katalas en, deren Wirkung nach Engler und $W$ eiß berg $^{2}$ ) auf dem vielleicht nur vorübergehenden, superoxydartigen Charakter beruht, der auf Peroxyde unter Reduktion und Entbindung von $\mathrm{O}_{2}$ wirkt. Durch die Zerstörung von Peroxyden werden Katalasen wohl zu Regulatoren der Oxydation im Organismus.

Schon Thénard, ${ }^{3}$ ) der Entdecker des Wasserstoffsuperoxyds, fand, daß Fibrin und tierisches Gewebe, ebenso wie Edelmetalle $\mathrm{H}_{2} \mathrm{O}_{2} \mathrm{zu}$ zersetzen vermögen. Von Schönbein ${ }^{4}$ ) wurden diese Studien noch weiter ausgedehnt und Bergengruen ${ }^{5}$ ) nahm bereits an, daß jedem Protoplasma die Fähigkeit zukäme, $\mathrm{H}_{2} \mathrm{O}_{2}$ zu zerlegen. Gottst ein ${ }^{6}$ ) führte die Erscheinung

2. T., S. 210, 242 usw.), an die Denitrifikation und an die Sulfatreduktion bis zu $\mathrm{NH}_{3}$ resp. $\mathrm{SH}_{2}$ erinnert [Beijerinck, Zentralbl. f. Bakt., II, Bd. I, S. 1 (1895)]. Auch die Buttersäuregärung, Sumpfgasgärung und andere komplizierte Vorgänge wären hier zu erwähnen. Heffter (Archiv für exp. Path., Festschr. f. Schmiedeberg, 1908, S. 253) hat gezeigt, daß gewisse von den genannten Reaktionen durch Extrakte aus dem Tierkörper hervorgerufen werden können, wie die von Nitrat zu Nitrit, von Nitrokörpern zu Aminen. Er hat weiter gezeigt [Arch. f. exp. Pathol., 1901, Bd. XLVI, S. 230; Med.-nat. Arch., Bd. I, S. 81 (1907)], daß manche der angeführten Reaktionen, wie die Reduktion der Arsensäure, von Jodat, Farbstoffen, Nitrobenzol, u. a. durch Su lfh y dr y lverbindungen, wie Cystein, erhalten werden.

1) E. und W., S. 47.

₹) Das., S. 190.

3) Ann. chim. et phys., Bd. IX, S. 315 (1818); Bd. XI, S. 86 (1819).

4) Journ. f. prakt. Chem., Bd. LXXXIX, S. 328 (1863).

s) Diss. Dorpat, 1888; Ghem. Zentralbl., 1889, Bd. I, S. 545.

6) Virchows Arch., Bd. CXXXIII, S. 295 (1893). 
auf die Zellnucleine zurück. Es scheint, daß Raudnitz ${ }^{1}$ ) zuerst als Ursache des Umsatzes ein Ferment annahm. Wichtig war die Arbeit Jacobsons, ${ }^{2}$ ) durch die die aufgetauchte Meinung widerlegt wurde, jedem Ferment käme die Fähigkeit der $\mathrm{H}_{2} \mathrm{O}_{2}$ Zerlegung an sich zu, und die sie auf ein spezifisches Enzym zurückführte. O. Loew ${ }^{3}$ ) hat dann auf das stetige Vorkommen solcher Fermente hingewiesen und ihnen den Namen Katalase gegeben.

In der Tat scheinen die Katalasen niemals den Organismen zu feblen und sie lassen sich in Organextrakten, Filtraten, in konservierten Zellen usw. nachweisen. So wurde der Beweis für Anwesenheit geliefert in Bakterienfiltraten (Gottstein, ${ }^{4}$ ) Löwenstein), ${ }^{5}$ ) in höheren Pilzen (Hefe: Jssajew $\left.{ }^{6}\right)$ PozziEscot, ${ }^{7}$ ) Henneberg, ${ }^{8}$ ) Boletus scaber: Euler, ${ }^{9}$ ) vgl. auch Loew $) ;{ }^{10}$ ) ferner in den Blättern von Tabak (Loew) ${ }^{10}$ ) und Tee (Aso und Pozzi-Escot), ${ }^{11}$ ) in Kürbisfrüchten und Meerettigwurzeln (Bach und Chodat), ${ }^{12}$ ) in der Milch (Babcock, ${ }^{13}$ ) Raudnitz, ${ }^{14}$ ) Loew, ${ }^{10}$ ) Jolles, ${ }^{15}$ ) Faitelowitz, ${ }^{16}$ ) E. Reiß ${ }^{17}$ )

1) Zentralbl. f. Physiol., Bd. XII, S. 790 (1899); Z. f. Biol., Bd. XL, S. 91 (1901); Bd. XLII, S. 102 (1902).

?) Diese Zeitschrift, Bd. XVI, S. 340 (1892).

3) Rep. Agric. Depart. Washington, Nr. 68(1901); Z. f. Biol., Bd. XLIII, S. 256 (1902); Zentralbl. f. Bakt. (II), Bd. X, S. 177 (1903); B. B., Bd. XXXV, S. 2487 (1902); «Chem. Energie der lebenden Zelle», 2. Aufl. (1906).

4) 1. c.

5) Münch. Med. Wochenschr., Bd. L, Nr. 50 (1904).

6) Diese Zeitschrift, Bd. XLII, S. 102 (1904); Bd. XLIV, S. 546 (1905).

`) Bull. de l'Assoc. de chim. des buer. et dest., Bd. XXI, S. 1247 (1904).

8) Z. f. Spiritusind., Bd. XXVII (1904).

$\left.{ }^{9}\right)$ Hofmeisters Beitr., Bd. VII, S. 1 (1905).

10) 1. c.

11) Rev. gén. de chim. pour. et appl., Bd. V, S. 419 (1903).

12) B. B., Bd. XXXVI, S. 609 (1903).

13) Ann. Agr. Strt. Univ. Wisconsin, S. 63 (1889).

14) 1. c., s. auch Ergebnisse der Physiol., Bd. II, 1., S. 193 (1903); das. auch weitere Literatur; ferner J. H. Kastle u. Madison; B. Porch, Journ. of Biolog. Chem., Bd. IV, S. 301 (1908), und J. H. Kastle u. Norman Roberts, Art. 10, Bull. Nr. 41, Hygienic Laboratory (zit. das.).

${ }^{5}$ ) Z. f. Biol., Bd. XLV, S. 248 (1903).

16) Diss. Heidelberg, 1904.

17) Z. f. klin. Med., Bd. LVI, S. 1 (1905). 
u. a. usw.), in den Extrakten von Insekten, Spinnen, Scorpionen usw. (Kobert und Fischer), ${ }^{1}$ ) in den Geweben und Zellen der Wirbeltiere. ${ }^{2}$ )

Es ist nicht wahrscheinlich, daß eine in den Organismen so verbreitete Wirkung nur einer Substanz zukommt. So hat auch $\mathrm{Loew}^{3}$ ) bereits die Existenz wenigstens zweier Fermente, der in Wasser unlöslichen $\alpha$-Katalase und der löslichen $\beta$-Katalase, angenommen. Ähnlich ließen sich auch Versuche von van Laer, ${ }^{4}$ ) sowie die Befunde Eulers ${ }^{5}$ ) deuten.

Es sei noch erwähnt, daß die Katalase nicht mit den Oxygenasen identisch ist. ${ }^{6}$ ) Ebensowenig aber stören sich Katalase und Peroxydase. ${ }^{7}$ )

Die Versuche von Batelli und Stern ${ }^{8}$ ) machen ein Vorkommen positiver und negativer Katalysatoren im Organismus wahrscheinlich. Dieselben Verfasser ${ }^{9}$ ) suchen auch einen experimentellen Nachweis für die physiologische Bedeutung der Katalasen dadurch zu geben, daß diese imstande sind, die Oxydation durch Ferrosulfat zu mäßigen. $\mathrm{Ph}$. Shaffer ${ }^{10}$ ) hat beobachtet, daß das Vorkommen von Katalase die Oxydation durch Wasserstoffsuperoxyd, z. B. von Harnsäure hemmt. Es kann sich also hier um einen weiteren wichtigen Regulationsmechanismus des Organismus handeln.

1) Pflügers Arch., Bd. XCIX, S. 116 (1903).

2) Literatur s. in Czyhlarz und Fürth, l. c., und Lockemann, Thies und Wichern, Diese Zeitschrift, Bd. LVIII, S. 390 (1909).

3) Zentralbl. f. Bakt. (II), Bd. X, S. 177 (1903).

4) Bull. Soc. chim. de Belg., Bd. XIX, S. 337 (1905).

5) Arkiv för Kemi, Bd. I, S. 357 (1904). Die schon von Liebermann gemachte Beobachtung, daß das Fettgewebe besonders reich an Katalase ist, läßt sich wohl am plausibelsten mit Reiss. als Adsarption erklären. Vgl. dazu Zentralblatt f. Bakt. (II), Bd. XXI, Heft 1, S. 609 (1908).

6) B. B., Bd. XXXVI, S. 601 (1903).

7) Das., Bd. XXXIX, S. 1670 (1906).

9) C. R., Bd. CXE, S. 1197 (1905).

9) Das., Bd. CXLI, S. 1044 (1906).

10) Am. Journ. of Physiol., Bd. XIV, S. 299 (1905). 


\section{SchluB.}

Die Einwirkung des gasförmigen Sauerstoffs auf Verbindungen wird durch die Autoxydationstheorie umfaßt, mit anderen Worten: Wo es sich um eine Wirkung des gasförmigen Sauerstoffs handelt, ordnen sich die Erscheinungen den oben auseinandergesetzten, allgemein gültigen Schematen unter; es kann sich also um direkte oder indirekte Autoxydation handeln, unter gewissen Umständen muß sich Wasserstoffsuperoxyd bilden usw.

Da man weiß, daß die Organismen gasförmigen Sauerstoff aufnehmen und chemisch verarbeiten, muß daher die orste Phase der biologischen Wirkung des Sauerstoffs als Autoxydation (unter Bildung von Moloxyden) angesprochen werden und die sich dabei abspielenden chemischen Vorgänge sind dem allgemein gültigen Schema für dieselbe einzuordnen.

Die weiteren Oxydationsvorgänge, die Übertragung des Sauerstoffs von den Peroxyden auf die Acceptoren sind bisher nicht in annähernd ähnlich weitgehender Weise zu klassifizieren, nur einige allgemeine Einflüsse, wie der der Reaktionsgeschwindigkeit, sind bisher erkannt, im übrigen kann zumeist nur eine spezielle Analyse zur Erkenntnis führen.

Daß in dem behandelten biologischen Kapitel im Verhältnis zu anderen wenig erhebliche Fortschritte erzielt worden sind, dürfte demnach wohl darin seinen Grund haben, daß in jenem Teil der Oxydationsprozesse, wo der gesamte Vorgang übersichtlich ist, in den meisten Fällen das Wissen von den Stoffen - der physiologischen Autoxydatoren - fehlt, während die Kenntnis der viel komplizierteren Umwandlungen von Acceptoren eben nur sehr viel schwieriger zu einem Einblick in den Gesamtvorgang zu führen vermag.

Es erscheint somit als eine wichtige Aufgabe, jene autoxydablen Stoffe im Organismus genauer kennen zu lernen, deren Peroxyde als Oxydationsmittel zu wirken vermögen und wahrscheinlich mittels Hilfsstoffen und Hilfsreaktionen die physiologischen Oxydationen durchführen. Man hat sie sich wegen der ihnen notwendig eigentümlichen großen Reaktionsfähigkeit 
jedenfalls in bestimmter Weise als sehr labil vorzustellen. Daß die Stoffe den bisher bekannt gewordenen Oxydasen anzugliedern sind, ist sehr wahrscheinlich, wenn auch noch keineswegs sicher. Jedenfalls wäre es völlig verfrüht, sie unter die Fermente zu reihen; ist doch schon die Unterordnung der als Oxydasen bezeichneten Agenzien unter die echten Fermente im Sinne von Katalysatoren vielleicht abzulehnen.

Wahrscheinlich lehrt im Gegenteil gerade das weitere Studium der physiologischen Oxydation, daß nicht den Fermentvorgängen allein die beherrschende Bedeutung zukommt, die ihnen heute von der biochemischen Forschung zugeschrieben wird, sondern daß als neue Momente die gekoppelten Reaktionen aufzusuchen sind, denen der Organismus auch den ihm eigentümlichen Charakter des chemischen Regulationsmechanismus verdankt. 